\title{
The role of surfactant type and bubble surface mobility in foam rheology
}

\author{
Nikolai D. Denkov, ${ }^{* a}$ Slavka Tcholakova, ${ }^{a}$ Konstantin Golemanov, $^{a}$ K. P. Ananthpadmanabhan ${ }^{b}$ and Alex Lips ${ }^{c}$ \\ Received 20th February 2009, Accepted 14th May 2009 \\ First published as an Advance Article on the web 14th July 2009 \\ DOI: 10.1039/b903586a
}

This paper is an overview of our recent understanding of the effects of surfactant type and bubble surface mobility on foam rheological properties. The focus is on the viscous friction between bubbles in steadily sheared foams, as well as between bubbles and confining solid wall. Large set of experimental results is reviewed to demonstrate that two qualitatively different classes of surfactants can be clearly distinguished. The first class is represented by the typical synthetic surfactants (such as sodium dodecylsulfate) which are characterised with low surface modulus and fast relaxation of the surface tension after a rapid change of surface area. In contrast, the second class of surfactants exhibits high surface modulus and relatively slow relaxation of the surface tension. Typical examples for this class are the sodium and potassium salts of fatty acids (alkylcarboxylic acids), such as lauric and myristic acids. With respect to foam rheology, the second class of surfactants leads to significantly higher viscous stress and to different scaling laws of the shear stress vs. shear rate in flowing foams. The reasons for these differences are discussed from the viewpoint of the mechanisms of viscous dissipation of energy in sheared foams and the respective theoretical models. The process of bubble breakup in sheared foams (determining the final bubble-size distribution after foam shearing) is also discussed, because the experimental results and their analysis show that this phenomenon is controlled by foam rheological properties.

\section{Introduction}

The dispersion of bubbles into surfactant solution, with an air volume fraction $\Phi=V_{\text {air }} / V_{\text {foam }}$ higher than the volume fraction

${ }^{a}$ Laboratory of Chemical Physics \& Engineering, Faculty of Chemistry, Sofia University, 1 James Bourchier Ave.,1164, Sofia, Bulgaria. E-mail: ND@LCPE.UNI-SOFIA.BG; Fax: (+359-2) 962 5643; Tel: (+359-2) 9625310

${ }^{b}$ Unilever Global Research Center, Trumbull, Connecticut, 06611, USA

'Unilever Discover, Port Sunlight Laboratory, Quarry Road East Bebington, CH63 3JW Wirral, UK of closely packed spheres, $\Phi_{\mathrm{CP}}$, leads to the formation of foams in which the bubbles are separated by thin, plane-parallel films, stabilized by surfactant adsorption layers. This process leads to a remarkable transformation of two Newtonian fluids (air and water) into a complex fluid with nontrivial visco-elastoplastic rheological properties, ${ }^{1-6}$ see Fig. 1. At low shear stress, the foams deform without flowing (at least in short time-scales) and this response could be described by elastic modulus, like those used to characterize the deformation of solids. At a certain "yield" stress, foams undergo "solid-to-fluid" plastic transition, above which they flow as shear-thinning fluids, with their effective viscosity being a decreasing function of shear rate.

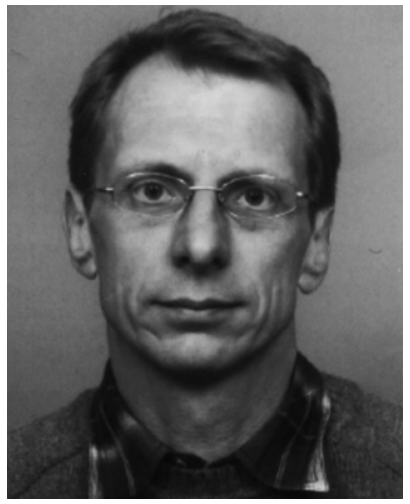

Nikolai D. Denkov
Nikolai Denkov was born in Bulgaria in 1962. He received his PhD (1993) and DSc (2007) in physical and theoretical chemistry from Sofia University, Bulgaria. His career is linked to Laboratory of Chemical Physics \& Engineering (LCPE) in the Faculty of Chemistry of same University, with sabbatical years in the institutes of JRDC (Japan, 1994), Rhodia (France, 1997); and Unilever (USA, 2004). His research includes studies on formation, stability, and rheology of dispersions (mainly foams and emulsions) and methods for their characterization (thin liquid films, light scattering, rheometry). He has published 106 research and review articles, cited 2700 times in literature.

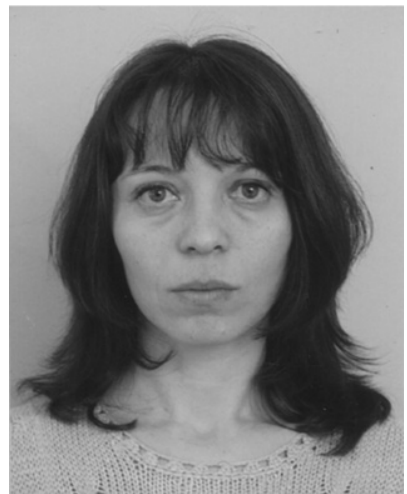

Slavka Tcholakova
Slavka Tcholakova was born in Bulgaria in 1972. She received PhD in Physical Chemistry (2004), and became Assistant Professor (2006) and Associate Professor (2009) in the Faculty of Chemistry, Sofia University, Bulgaria. She has been visiting researcher in the Research Center Paul Pascal, CNRS, Bordeaux, France (1997). Main research interests: formation, stability and rheology of emulsions; protein adsorption in relation to emulsion stability; and foam stability in the presence of antifoams. She has published 35 research and review articles, cited over 250 times. She was the recipient of the University of Sofia award for "Best Young Scientist" for 2006. 
During the past several years, there has been a rapidly increasing interest in several scientific areas of this complex rheological behaviour of foams, because it represents in many aspects the behaviour of a wide variety of dispersions of soft particles, such as drops, microgel beads, lipid vesicles, and others. ${ }^{2,4}$ Foams seem particularly suitable for studying and modelling the dynamic phenomena in such complex systems (e.g., yield transition, linear visco-elasticity, non-homogeneous flow, etc.), because the foam behaviour is governed by relatively well understood interplay of capillary effects and viscous friction in the foam films, formed between neighbouring bubbles. ${ }^{1,6-18}$ In addition, the relatively large bubble size allows direct optical observation of the dynamics of individual bubbles in flowing foams. ${ }^{18-21}$ All these circumstances provide unique possibility for detailed theoretical modelling and experimental studies of foams at a microstructural level, which is impossible for the other systems of interest.

In the course of these studies, the researchers have formulated adequate explanations and quantitatively described several of the

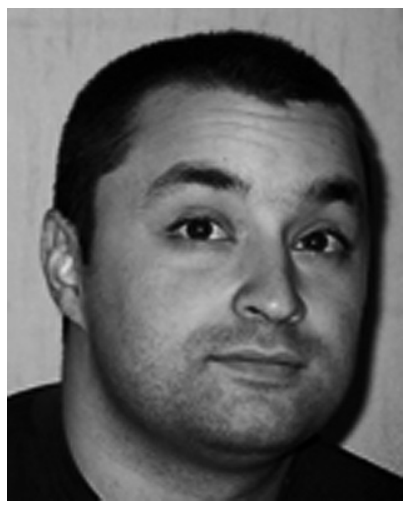

Konstantin Golemanov

cited more than 70 times in literature.
Konstantin Golemanov was born in Bulgaria in 1981. He received his BSc (2002) and PhD (2009) in physical and theoretical chemistry from Sofia University, Bulgaria. He is currently a research associate at the Laboratory of Chemical Physics \& Engineering ( $L C P E)$ in the Faculty of Chemistry of the same University. His research interests include rheology of foams and emulsions, and Pickering emulsions. He is a coauthor of 9 research articles,
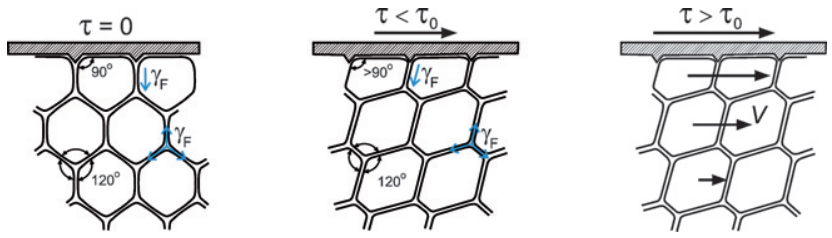

Fig. 1 A schematic presentation of the origin of elastic and viscous responses of foam subject to shear stress, $\tau$. (A) In absence of external stress, the bubbles are symmetrical and the tensions of the foam films, $\gamma_{F}$, are balanced both inside the foam and at the confining solid wall. (B) At low external stress, the bubbles deform and the resulting slope of the foam films creates elastic stress inside the foam and in the contact zone foam-wall. Due to the preserved static angles between the foam films, $120^{\circ}$, the forces acting on each bubble are again balanced, despite bubble deformation. (C) At external stress higher than the foam yield stress, $\tau>$ $\tau_{0}$, the static balance is lost and the bubbles are forced to slide along each other in the flowing foam. The viscous friction in the foam films, formed between neighbouring bubbles which move with different velocities, leads to energy dissipation and, hence, to appearance of foam viscous stress, $\tau_{V}$.

basic dynamic properties of foams. The elastic behaviour of foams was explained with capillary effects, which appear as a result of bubble deformation and resulting inclination of the foam films in direction of the applied external stress, see Fig. 1B. Because each foam film bears a mechanical tension approximately equal to the doubled surface tension of the liquid $\left(\gamma_{\mathrm{F}} \approx\right.$ $2 \sigma$ ), this film inclination builds a mechanical stress in the foam, which balances the external stress, and thus prevents the ensemble of bubbles from flowing, see Fig. 1B. Note that for each bubble inside the static foams, the film stresses are balanced so that (despite being deformed) the bubbles are trapped in a selfsupporting static structure. In the same line of considerations, the yield transition was explained ${ }^{1,8}$ as the moment when the bubbles deform so much that the balance of film tensions is lost and the bubbles are forced to begin sliding along each other, Fig. 1C. This sliding leads to viscous friction in the foam films separating the neighbouring bubbles and to appearance of the respective foam viscous stress.

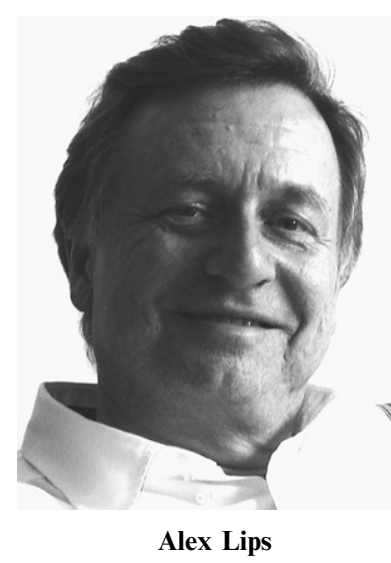

Alex Lips was born in Austria in Kavssery P. Ananthapadmanabhan (Ananth) was born in Kerala, India in 1952. He received his $B$. Tech. degree from Indian Institute of Technology, Mumbai, India and his Masters (1976) and Doctorate (1980) from Columbia University in New York. He was a member of the Surface Chemistry Skill Center of Union Carbide Corporation from 1983 to 1990. He joined Unilever $R \&$ $D$ in 1990 where he is now a lead scientist leading a group on surfactants and polymers. His research interests include surfactant solution chemistry, surfactant interactions with polymers, proteins, bio-membranes and skin.
1946. He received his BSc degree in chemistry from Imperial College, London in 1968, and he embarked on his long career in Unilever Research in that year. In 1974 he received his Ph.D. degree in Colloidal Light Scattering. He has been researching in a wide range of areas concerning home and personal care as well as foods. His research interests include colloidal optics and rheology, surfactants, polymers and emulsions. He is currently the Discover Director for Materials at Unilever Discover Port Sunlight Laboratory, UK. 
The performed experimental studies demonstrated that, along with their general patterns of dynamic behavior, foams exhibit some strong system-specific effects, which are still poorly understood. ${ }^{17,22-25}$ These specific effects are in most cases related to the type of foam stabilizers used (surfactants, polymers, particles). Despite the importance of this specificity for the ubiquitous practical applications of foams, it often creates serious problems in the scientific interpretation of the experimental data, in the comparison of the results obtained by different groups, and in the generalization of the obtained results and conclusions to other related systems. Therefore, the major goal of this paper is to present an overview of our current understanding of the effects of surfactant type on foam rheological properties, with focus on the viscous friction in foams, for which the surfactant-specific effects are most pronounced.

For first time the role of surfactant type in foam dynamics was demonstrated convincingly in the studies of foam film drainage. ${ }^{10,11,22,26-30}$ In some of these early studies, foam films stabilized by mixtures of sodium dodecylsulfate (SDS) and lauryl alcohol $(\mathrm{LOH})$ were shown to thin down in a regular manner, with a gradual change of their thickness along the film area. ${ }^{26-28}$ This is in sharp contrast with the films stabilized by typical synthetic surfactants (such as pure SDS without cosurfactants) which thin down with formation of irregular dynamic eddies in the so-called "marginal regeneration zone" at the film periphery. ${ }^{29,31}$ This qualitative difference in the modes of film thinning was explained with the formation of surface condensed phase in the mixed surfactant adsorption layer of SDS and $\mathrm{LOH} .{ }^{26}$ This condensed phase is presumably providing "rigidity" of the solution surface, thus affecting the liquid flow inside the foam film, by controlling the boundary conditions at the film surfaces..$^{11-14,32}$

More recently, the effect of surfactant type was demonstrated in the studies of liquid drainage from foams. ${ }^{33-45}$ The experiments and theoretical analysis showed that, depending on surfactants used, the walls of the Plateau borders could be considered as tangentially mobile or immobile, which has a pronounced effect on the regime of liquid drainage. For ensuring different surface mobility, either solutions of different commercial liquid detergents were used (presumably with different surface properties) $)^{33,38}$ or lauryl alcohol ( $\left.\mathrm{LOH}\right)$ was added as a cosurfactant to SDS or tetradecyl-trimethylammonium bromide (TTAB) solutions to render surface "rigidity". ${ }^{34,38,42-45}$

Obviously, the dynamic properties of bubble surfaces could be important for all dynamic phenomena in foams, including foam flow, foam expansion in the process of extrusion (e.g., in cosmetic products, such as shaving and hair foams; and during production of plastic foams from liquid precursors), Ostwald ripening (important for long-term stability of food and cosmetic products), dispersion of particles in the network of Plateau channels (relevant to froth flotation and waste water treatment), and many others. ${ }^{2-5}$ However, the studies focused on the role of surfactant type on these phenomena were very limited until recently.

During the past several years, our group performed a series of related experimental and theoretical studies which aimed to clarify the role of bubble surface mobility in foam rheology. ${ }^{16-18,21,23-24,46}$ The accumulated results have allowed us to demonstrate several significant effects of surfactant type on foam dynamics and to provide theoretical explanations for some of these effects. In parallel, the effects of surfactants on dynamic phenomena in foams were studied by several other research groups..$^{25,47-50}$ In the current review we summarise the progress achieved so far in these studies. Comparison with the results of the various research groups is made, and some open questions, deserving further investigation, are discussed.

The article is structured as follows: in section 2 we illustrate with experimental results the effect of surfactant type on foam rheological properties, related to viscous friction in foams and to foam-wall friction. In section 3 we demonstrate how one can modify bubble surface properties by selection of appropriate cosurfactants (note that term "cosurfactant" refers to a surfactant which is added in much lower concentration in the solution, compared to the main surfactants, while being able to affect significantly surface and foam properties). In section 4 we explain the theoretical basis of the observed relation between bubble surface mobility and foam rheological properties. In section 5 we make a comparison between the results obtained by the various research groups. In section 6 we present illustrative results of the effect of surfactant type on the process of bubble breakup in sheared foams and explain these results by the different rheological properties of the foams. The main conclusions are summarized in section 7 .

\section{Experimental results demonstrating the role of surfactant type in foam rheology}

In this section we summarize a large set of experimental results, ${ }^{21,23,24}$ which demonstrate the effect of surfactant type on the viscous friction inside sheared foam, and between foam and confining solid wall (foam-wall friction).

\subsection{Inside-foam viscous friction}

As a rule, the measured shear stress with foams is very well represented by Herschel-Bulkley equation (see Fig. 2A): 1,9,17,23,25

$$
\tau(\dot{\gamma})=\tau_{0}+\tau_{\nu}(\dot{\gamma})=\tau_{0}+k \dot{\gamma}^{n}
$$

which includes three parameters: yield stress $\tau_{0}$, power-law index $n$, and consistency, $k$. Here $\tau_{V}(\dot{\gamma})$ is the rate-dependent fraction of the total stress, which is determined by subtraction of the constant term, $\tau_{0}$, from the total stress, $\tau(\dot{\gamma})$. The power-law index, $n$, is a characteristic of the shear-thinning behaviour of the system. For Newtonian liquids $n=1$, whereas for shear-thinning fluid $n<1$. For foams and concentrated emulsions with $\Phi>\Phi_{\mathrm{CP}}$, values of $n$ between 0.2 and 0.5 are usually reported., ${ }^{9,1723,25}$ The interpretation of the values of the consistency $k$ is more complex, because the dimension of $k$ is $\mathrm{Pa} . \mathrm{s}^{\mathrm{n}}$ and, therefore, it depends on the specific value of $n$, see eqn (1). The simplest case is $n=1$, when $k$ coincides with the common viscosity, as defined for Newtonian fluids.

The dependence of the yield stress, $\tau_{0}$, on the foam (or emulsion) characteristics was studied in detail both theoretically and experimentally, and the main experimental results have been already explained by considering the static characteristics of the foams, such as the equilibrium surface tension, $\sigma$, mean volumesurface radius, $R_{32}$, bubble polydispersity, and bubble volume fraction, $\Phi .^{1,4,6-9,51-53}$ Therefore, the yield stress is not considered 

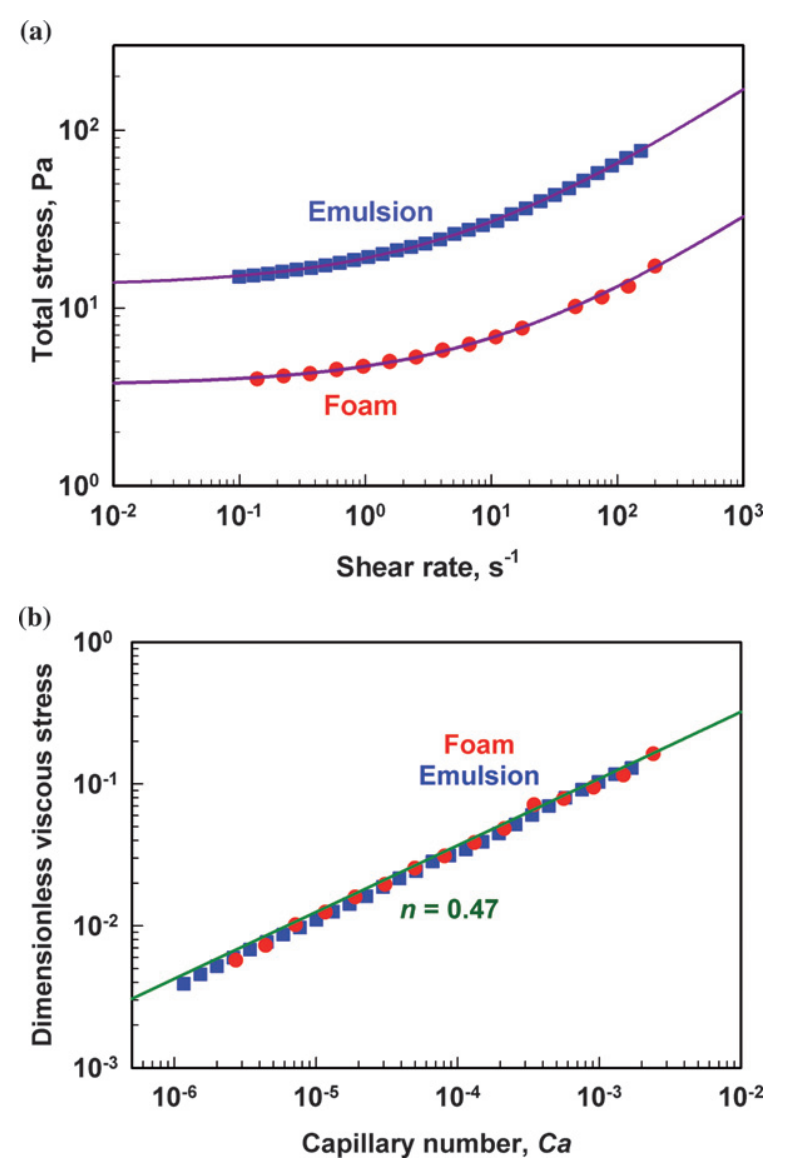

Fig. 2 Illustrative experimental results, demonstrating the convergence of the data for foams and emulsions, when approproiate dimensionless plot is used. (A) Total stress, $\tau$, as a function of shear rate, $\dot{\gamma}$, for hexadecane-in-water emulsion stabilized by nonionic surfactant $\left(R_{32}=4 \mu \mathrm{m}\right.$, $\sigma=2 \mathrm{mN} / \mathrm{m}, \mu=5 \mathrm{mPa}$.s blue squares) and foam stabilized by a mixture of anionic and amphoteric surfactants $\operatorname{SLES}+\operatorname{CAPB}\left(R_{32}=300 \mu \mathrm{m}, \sigma=\right.$ $30 \mathrm{mN} / \mathrm{m}, \mu=1 \mathrm{mPa} . \mathrm{s}$, red circles). The curves are best fits by the Hershel-Berkley model, eqn (1). (B) Dimensionless viscous stress, $\tilde{\tau}_{V}=$ $\tau_{V} R_{32} / \sigma$, as a function of the capillary number, $C a=\left(\mu \dot{\gamma} R_{32}\right) / \sigma$, for the same samples. The line represents theoretical result, eqn (5), which is plotted without any adjustable parameters. ${ }^{16,17}$ For both samples the volume fraction of the dispersed phase is $\Phi=0.90$.

here and the focus of the current review is on the rate-dependent component, $\tau_{\nu}(\dot{\gamma})$, which depends on the dynamic characteristics of the surfactant adsorption layers.

For comparison of the experimental results obtained with different foams and emulsions, it is convenient to use appropriate dimensionless quantities. The viscous stress can be scaled by the mean capillary pressure of the bubbles (drops) in the sample, $\tilde{\tau}_{V}=\tau_{V} R_{32} / \sigma$. This scaling was first introduced by Princen ${ }^{1,9}$ without a rigorous theoretical basis, by analogy with the scaling of the elastic modulus and osmotic pressure of concentrated emulsions and foams (for the latter two quantities this scaling follows directly from theoretical analysis ${ }^{1}$ ). Our recent theoretical model of the viscous friction in sheared foams and concentrated emulsions ${ }^{16,17}$ shows that the scaling of $\tau_{V}$ by $\left(\sigma / R_{32}\right)$ can be deduced from the theoretical expressions for the rate of energy dissipation in these systems (see section 4.1 below), thus providing a firm theoretical basis for this scaling.
As usual in the rheology of foams and emulsions, the shear rate is scaled by balancing the viscous stress and bubble capillary pressure, leading to the so-called "capillary number", $\mathrm{Ca}=$ $\left(\mu \dot{\gamma} R_{32}\right) / \sigma$, which plays the role of dimensionless shear rate in this context ( $\mu$ is solution viscosity).

Therefore, the experimental and theoretical results for the viscous stress are conveniently presented in dimensionless form as plots of $\tilde{\tau}_{V}$ versus $\mathrm{Ca}$. Such plots eliminate the generic effects of $R_{32}, \sigma$, and $\mu$, and allow one to make a direct comparison of results obtained with various emulsions and foams, clarifying the specific effects of the type of dispersion (foam or emulsion), surfactant type, and other non-trivial factors.

The usefulness of this way of data representation is demonstrated in Fig. 2, where the experimental data for $\tau(\dot{\gamma})$ for concentrated emulsion stabilized by nonionic surfactant $\mathrm{C}_{13} \mathrm{EO}_{10}$ are shown Fig. 2A to be much higher than the data obtained with foam, stabilized by a mixture of anionic and amphoteric surfactants (SLES: sodium lauryl-oxyethylenesulfate, and CAPB: cocoamidopropylbetaine). Remarkably, when the same experimental data are plotted in dimensionless form, the results for the viscous stress of the two systems merge to a single master line, see Fig. 2B. This agreement of the results obtained with foam and emulsion, which are so different in their material characteristics, unambiguously shows that the mechanism of viscous dissipation in these two particular samples is the same and could be described by a single theoretical model. In fact, we found that the same master line, representing eqn (5) below, describes very well the experimental data for numerous foams and emulsions with different values of $R_{32}, \sigma$, and $\mu$, for which the power-law index $n \approx 1 / 2$. Further experimental results are presented in ref. 21 and 23 and the respective theoretical explanation is given in section 4.1.1.

Not all foams and emulsions obey the same power-law index, however. The dimensionless plots $\tilde{\tau}_{V}$ vs. $\mathrm{Ca}$ have revealed two qualitatively different types of systems (all of them selected to have Newtonian behavior of the continuous phase), see Fig. 3:

(1) Systems in which the power-law index $n \approx$ $1 / 2{ }^{9,16,17,23-25,54,55}$ In these systems $n$ varied between 0.42 and 0.5 (centred at $n \approx 0.47$ ), depending on the specific surfactant and glycerol concentration in the foaming solution.

(2) Systems with significantly lower value of the index, $n<1 / 2$; for most of these systems $n$ is between 0.20 and $0.25 .^{17,23-25,56}$ Systems of the second type exhibit much higher viscous friction at similar material characteristics $\left(\Phi, \mu, \sigma\right.$, and $\left.R_{32}\right)$, as compared to the first type of systems. ${ }^{23,24}$

In the literature, one can find data described with $n>$ $1 / 2,{ }^{49,50,52,57-59}$ however, these results are typically obtained at lower volume fractions, $\Phi<0.75$, and will not be considered in detail, because the focus of our study is on the concentrated emulsions and foams $(\Phi \geq 0.80)$. A short discussion of the systems with $\Phi<0.75$ is presented in section 5.1.

To understand the reasons for this qualitatively different behaviour of the foams stabilized by various surfactants, we have characterized the dynamic surface properties of the foaming solutions. ${ }^{23,24}$ These experiments showed that the rheological behaviour of the foams correlates with the surface dilatational modulus, $E_{\mathrm{D}}$, which is a quantity characterizing the amplitude of variation of the surface tension of the solution, $\sigma$, upon sinusoidal perturbation of the solution surface area (see section 3.1 

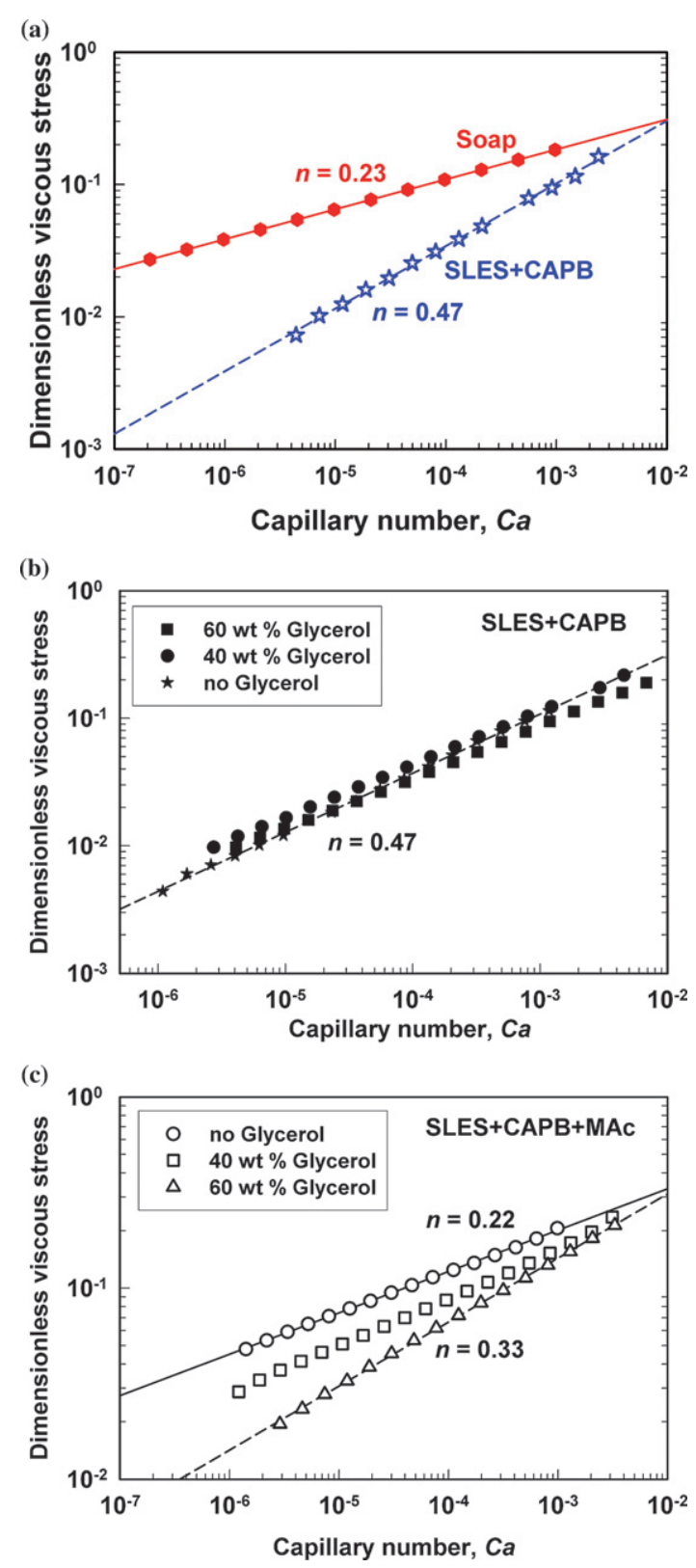

Fig. 3 (A) Illustrative experimental results, demonstrating the role of surfactant type for the viscous stress in sheared foams. (B) Dimensionless viscous stress as a function of capillary number for foam stabilized by SLES + CAPB at different concentrations of glycerol (0, 40, $60 \mathrm{wt} \%)$, used to vary solution viscosity. (C) Dimensionless viscous stress as a function of capillary number for foam stabilized by SLES + CAPB + MAc at different glycerol concentrations. Air volume fraction is $\Phi=$ 0.90 . The lines are drawn as eye-guides with the slope index, $n$, as indicated on the figures.

for further explanations). All systems of the first type, with $n \approx$ $1 / 2$, are characterized by low values of $E_{\mathrm{D}} \cdot{ }^{23,24}$ For these systems, the dimensionless numerical pre-factor in the dependence $\tilde{\tau}_{V}(C a)$, defined as $\tilde{k}=\left(k R_{32}^{1-\mathrm{n}}\right) /(\sigma \mu)^{1-\mathrm{n}}$, is practically independent of $\mu, \sigma$, and $R_{32}$ (see Fig. 3B for example), and depends explicitly on the volume fraction of the dispersed phase only. For these systems, the increase of $\Phi$ leads to higher value of $\tilde{k}$, while the power-law index remains virtually the same, $n \approx 1 / 2 .{ }^{23,25}$
Measurements of the surface properties showed that the foams of the second type are stabilized by surfactants rendering high surface modulus and relatively long relaxation times after surface perturbation (seconds and dozens of seconds). ${ }^{23-25}$ Examples for such surfactants are the sodium and potassium laurates, myristates, palmitates, cocoylglycinates, etc. For these systems, the value of $n$ is around 0.2 (at $\mu \approx 1 \mathrm{mPa} . \mathrm{s}$ ) and the measured viscous stress does not scale with solution viscosity, see Fig. 3C for example. Furthermore, the power-law index, $n$, changes its value upon variations of $\Phi$ and/or $\mu$ (the respective explanation is given in section 4.1 .2 below). ${ }^{23}$

These results show clearly that the mechanisms of viscous dissipation in foams, stabilized by surfactants with low surface modulus (LSM) and by surfactants with high surface modulus (HSM), are different. These mechanisms are discussed in section 4 below.

\subsection{Foam-wall viscous friction}

If the foam is in contact with smooth solid wall, the application of external stress usually leads to sliding of the bubbles along the wall surface, thus violating the usual "non-slip" boundary condition for fluid flow at solid surfaces. ${ }^{1-3,6,8,23,25}$ This "foam slip" phenomenon has an important effect on the overall foam rheology, because it changes the relationship between the applied external stress and the actual shear rate inside foam. Foam slip is conveniently studied by placing the foam in contact with solid surface in the rheometer (e.g., with the wall of a Couette cylinder, plate, or cone) and applying an external stress that is lower than the yield stress of the foam. ${ }^{8,23,25}$ Under these conditions, a foamwall slip at controlled relative velocity is realized and the measured shear stress is due entirely to the viscous friction of the boundary layer of foam bubbles with the smooth surface of the wall. Interestingly, very similar type of viscous friction is realized when a bubble or drop is travelling along narrow capillary tube, which presents another challenging research problem (often called "Bretherton problem"), related to practically important processes, such as tertiary oil recovery by surfactant solutions and microfluidics. ${ }^{3,6,60-66}$

As shown theoretically by Bretherton, ${ }^{60}$ Schwarz et al. ${ }^{61}$ and other authors, ${ }^{23,62-67}$ it is appropriate to scale the measured wall stress in these systems, $\tau_{W}$, by the bubble capillary pressure, $\tilde{\tau}_{W}=$ $\tau_{W} R_{32} / \sigma$, while the velocity of the moving plate, $V_{0}$, is scaled by $\sigma / \mu$, which again corresponds to a balance of the bubble capillary pressure and the viscous stress in bubble-wall friction. The latter scaling defines another dimensionless capillary number, $\mathrm{Ca}^{*}=$ $\mu V_{0} / \sigma$, which plays the role of dimensionless velocity in the description of bubble-wall or drop-wall friction. ${ }^{23,60-67}$

Similarly to the inside-foam viscous stress (section 2.1), the experimental results for $\tilde{\tau}_{W}$ vs. $\mathrm{Ca}^{*}$, measured with many different foams (with different mean bubble sizes, and prepared from various surfactant solutions with different viscosities and surface tensions) merged into two master lines, depending on the surface modulus of the foaming solutions, see Fig. 4: (1) systems with low surface modulus, corresponding to power-law index $m$ $\approx 2 / 3$; (2) systems with high surface modulus, corresponding to $m \approx 1 / 2 .{ }^{23-25,46}$ In this case, the index $m$ characterizes the observed power-law dependence, $\tilde{\tau}_{W}=k_{\mathrm{W}}\left(\mathrm{Ca}^{*}\right)^{\mathrm{m}}$. Further examples for these two types of systems are given in section 3 and the respective mechanistic explanation is discussed in section 4 . 


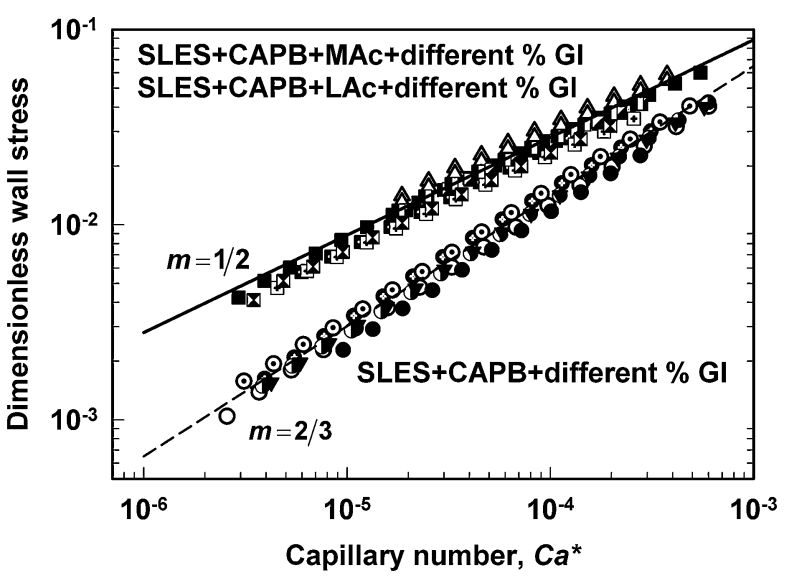

Fig. 4 Dimensionless foam-wall friction stress, $\tau_{W} /\left(\sigma / R_{32}\right)$, versus capillary number, $\mathrm{Ca}^{*}=\mu V_{0} / \sigma$, for foams prepared from different surfactant solutions. The viscosity of the foaming solutions was varied from 1 to $11 \mathrm{mPa}$.s by adding glycerol. Air volume fraction is $\Phi=0.90$. The lines are drawn as eye-guides with slopes corresponding to the indicated flow index, $m$.

Note that for foam-wall friction, the effect of solution viscosity is always properly accounted by using the dimensionless plot $\tilde{\tau}_{W}$ vs. $C a^{*}$ and all experimental data merge into two master lines. ${ }^{23,24}$ In contrast, for the inside-foam friction, in the case of HSM surfactants, the data obtained with solutions of different viscosities do not merge onto a single master line, see Fig. 3C. These results are explained by the theoretical models, developed in ref. 16 and 17 and briefly summarized in section 4.1 below.

\section{Control of surface mobility by choosing appropriate cosurfactants}

The experimental results presented in section 2 demonstrate clearly that two classes of surfactants can be distinguished, which differ significantly in the magnitude of the dilatational modulus of the respective surfactant solutions. ${ }^{17,23,24}$ As a result, these surfactants lead to qualitatively different regimes of foam-wall and inside-foam viscous friction: at low surface modulus (LSM) the power-law indexes $n \approx 1 / 2$ and $m \approx 2 / 3$, whereas at high surface modulus (HSM) $n$ is in the range $0.20-0.25$ and $m \approx 1 / 2$. Also, the viscous stress in foams characterized by HSM is much higher than that in foams stabilized by LSM surfactants, under otherwise equivalent conditions (similar solution viscosities, interfacial tensions, mean bubble sizes, etc.). The accumulated experimental data show that the boundary between "low" and "high" surface modulus systems is somewhere between $c a$. 30 and $100 \mathrm{mN} / \mathrm{m}$, depending on the other material parameters of the system. ${ }^{17,23,24}$ Table 1 summarizes the various possible cases, which are considered in the current review, and the respective explanations described in section 4 below.

For detailed systematic studies of the role of surface properties in foam dynamics, one should ideally have a range of surfactants providing: (1) a relatively wide range of reproducible and stable with time surface properties; (2) Newtonian behavior of clear foaming solution (without precipitates), with a possibility for wide variation of the solution's bulk viscosity; (3) very stable foams with suppressed bubble coalescence.

Most of the typical synthetic surfactants (SDS, SLES, CAPB) and their mixtures have solutions with Newtonian behaviour and relatively low surface modulus above the critical micelle concentration (CMC). On the other side, the alkycarboxylate (soap) solutions are characterized with very high surface modulus and, from this viewpoint, they complement the synthetic surfactants very well. ${ }^{23,68}$ However, soap solutions typically contain solid crystallites (precipitates) of complex composition, ${ }^{23,69,70}$ which may create problems in the experiments and data interpretation. These crystallites could be removed from the foaming solution by centrifugation or filtration, however, they spontaneously reappear for relatively short period of time (minutes or hours). Therefore, special procedures for preparation of the foaming solutions are required and relatively short experiments can be performed, if the experimentalist wants to avoid the presence of crystallites. ${ }^{23}$ Another related complication with soaps is that their solubility and surface rheological properties are strongly dependent on solution $\mathrm{pH}$, which cannot be controlled easily, because the dissolution of $\mathrm{CO}_{2}$ from the air and surfactant precipitation both lead to significant variations of the $\mathrm{pH}^{69,70}$

Using other foam stabilizers with relatively high surface modulus, such as proteins or lauryl alcohol as a cosurfactant to SDS and TTAB, also creates some technical problems when studying foam dynamics, which are discussed in ref. 24.

In the current section we explain how the accumulated knowledge of the role of surface properties could be used for

Table 1 Summary of the power-law indexes, $m$ and $n$, observed experimentally for foam-wall and inside-foam viscous friction (section 2 ), along with the proposed explanations for the observed values (section 4)

\begin{tabular}{|c|c|c|}
\hline Type of surfactant & $\begin{array}{l}\text { Power-law index for foam-wall } \\
\text { friction, } m\end{array}$ & $\begin{array}{l}\text { Power-law index for friction inside } \\
\text { foam, } n\end{array}$ \\
\hline Low-surface- modulus (LSM) & $\begin{array}{l}m \approx 2 / 3 \text { dominant friction in the } \\
\text { meniscus region around the } \\
\text { wetting film }\end{array}$ & $\begin{array}{l}n \approx 1 / 2 \text { dominant friction in foam } \\
\text { films }\end{array}$ \\
\hline High-surface- modulus (HSM) & $\begin{array}{l}m \approx 1 / 2 \text { dominant friction in the } \\
\text { wetting film } \\
1 / 2<m<2 / 3 \text { comparable } \\
\text { friction in the film and meniscus } \\
\text { regions }\end{array}$ & $\begin{array}{l}n \approx 0.2 \text { to } 0.3 \text { (increases with } \mu \text { ) } \\
\text { significant energy dissipation on } \\
\text { bubble surface }\end{array}$ \\
\hline Other factors & $\begin{array}{l}m<1 / 2 \text { shear-thinning foaming } \\
\text { solution or partial surface } \\
\text { mobility of bubbles }\end{array}$ & $\begin{array}{l}n>1 / 2 \text { low air volume fraction, } \Phi< \\
0.75\end{array}$ \\
\hline
\end{tabular}


designing a class of surfactant mixtures, which are appropriate for studying foam dynamics. First, we present illustrative results about the surface rheological properties of solutions, prepared with surfactants from the two different classes. Afterwards, these results are used as a reference to discuss the properties of the various surfactant mixtures which combine the advantages of the two surfactant types. ${ }^{24}$

\subsection{Surface rheological properties}

The surface dilatational rheological properties of the foaming solutions can be measured by the oscillating drop method (ODM) or in Langmuir trough..$^{22,71-74}$ In these methods, the surface of the surfactant solution is sinusoidally perturbed, $a(t)=$ $a_{0} \sin (\omega \mathrm{t})$, where $a(t)=\left[A(t)-A_{0}\right] / A_{0}$ is the normalized change of the surface area around the mean area, $A_{0}$, while $a_{0}$ is the relative amplitude of oscillations. The resulting variation of the surface tension is measured and (for small deformations) is presented as:

$$
\sigma(t)=E_{S D} a_{0} \sin (\omega t)+E_{L D} a_{0} \cos (\omega t)
$$

where $E_{\mathrm{SD}}$ is the surface storage modulus (related to surface elasticity) and $E_{\mathrm{LD}}$ is the surface loss modulus, which is related to surface dilatational viscosity, $\mu_{\mathrm{SD}}=E_{\mathrm{LD}} / \omega$. The total surface dilatational modulus is

$$
E_{D}=\left(E_{S D}^{2}+E_{L D}^{2}\right)^{1 / 2}
$$

The experiments show ${ }^{23,24}$ that high values of $E_{\mathrm{D}}$ are related to relatively long characteristic time, $t_{\sigma}$, for relaxation of the solution surface tension to its equilibrium value, after expansion or contraction of the surface. This relation originates in the fact that, for systems with long $t_{\sigma}$, the surface expansion (contraction) leads to significant variation of the amount of adsorbed surfactant, $\Gamma$, with resulting variation in $\sigma(\Gamma)$, without possibility for fast restoration of equilibrium adsorption by transfer of molecules toward (from) the surface via exchange with the underlying solution. In contrast, short relaxation times are related to fast exchange of surfactant molecules between the surface and the adjacent solution, so that the variation of surface area does not lead to significant changes in $\Gamma$ and $\sigma$.

As a representative for the surfactants with high surface modulus (HSM) we have chosen a mixture of several alkylcarboxylates (potassium salts of fatty acids) with different chain lengths, varied between 12 and 18 carbon atoms (from laurate to stearate). ${ }^{23}$ As a representative for the low-surface-modulus (LSM) surfactants we have chosen the mixture of SLES + CAPB. The major advantage of using this mixture, instead of single surfactants (such as SLES or SDS), is that it gives very stable foams and has large solubilization capacity for cosurfactants (many of them have low solubility in water). ${ }^{24}$ All experiments were performed with surfactant solutions having Newtonian behaviour (viscosity independent of shear rate) and with concentration above the CMC to ensure high foam stability in the related foam studies.

Illustrative results for $E_{\mathrm{D}}, E_{\mathrm{SD}}$, and $E_{\mathrm{LD}}$ of solutions, representing the two surfactant groups, are shown in Fig. 5. One sees that the differences between the two groups are very significant: in the HSM systems, the values of $E_{\mathrm{LD}}, E_{\mathrm{SD}}$, and $E_{\mathrm{D}}$ are about
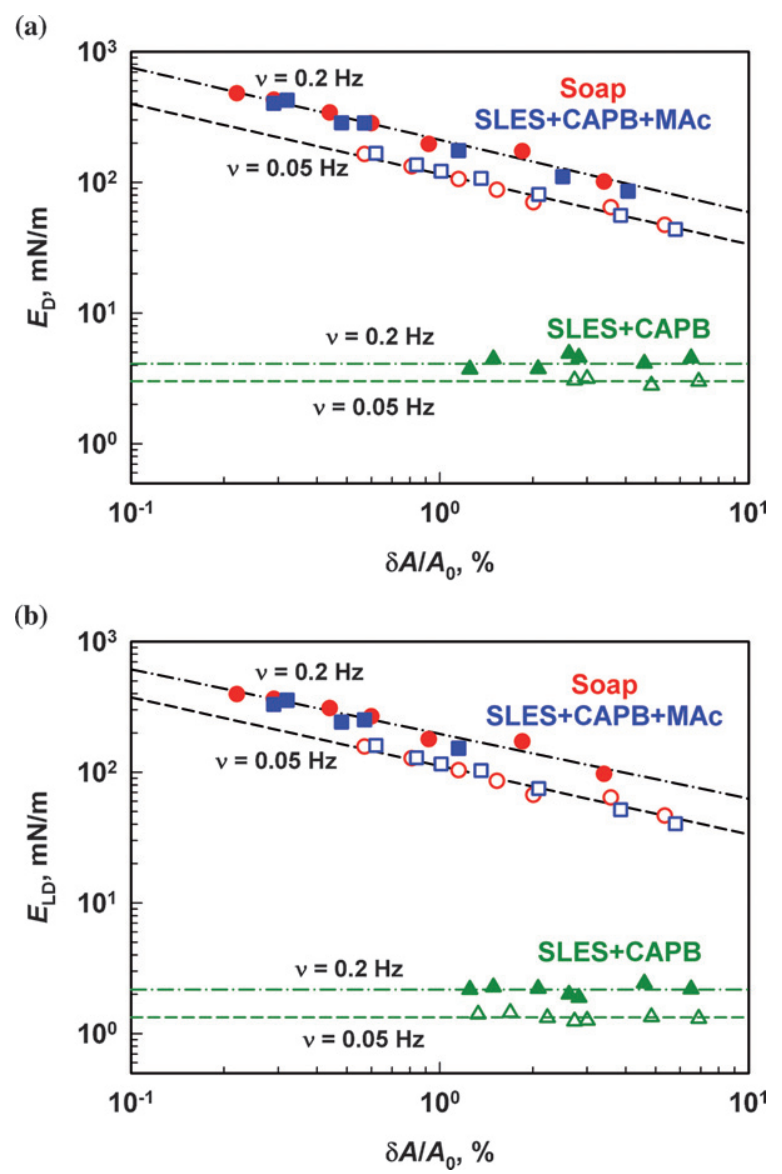

Fig. 5 (A) The total surface dilatational modulus, $E_{\mathrm{D}}$, and (B) the dilatational surface loss modulus, $E_{\mathrm{LD}}$, as functions of the relative surface deformation, $\delta A / A_{0}$, for soap (red circles), SLES + CAPB (green triangles), and SLES + CAPB + MAc (blue squares) solutions, measured by drop-shape analysis of oscillating pendant drops, at two frequencies of oscillations: $v=0.2 \mathrm{~Hz}$ (full symbols) and $v=0.05 \mathrm{~Hz}$ (empty symbols).

1-2 orders of magnitude higher than those for LSM systems and depend strongly on both the relative amplitude and frequency of oscillations. The strong dependence of the surface modulus on the oscillation amplitude shows that HSM systems are non-linear (even for very small amplitudes of the surface oscillations, $0.2 \%$ ), which implies that the molecular mechanism leading to the measured high moduli is non-trivial and is probably related to phase transitions in the adsorption layer, see the end of this section. In contrast, the values of the different moduli for the LSM systems are practically independent of the amplitude of oscillations (while depending on frequency), i.e. these are linear systems.

As explained in ref. 24, we could combine the advantages of the HSM and LSM systems by addition of a small amount of alkylcarboxylic acids (e.g., lauric, myristic, or palmitic) as a cosurfactant to the mixture of SLES + CAPB. These acids are solubilized in the surfactant micelles, which leads to formation of clear solutions with Newtonian behaviour. On the other hand, the alkylcarboxylic acids are more surface active and dominate the solution surface properties, thus rendering these solutions with a high surface modulus. 
Experimental data illustrating this concept are shown in Fig. 5 for the surfactant mixture SLES + CAPB + myristic acid (MAc). The concentration of MAc in the solution is $0.02 \mathrm{wt} \%$ only, whereas the other two surfactants are with much higher concentration ( 0.33 and $0.17 \mathrm{wt} \%$, respectively). One sees from Fig. 5 that $E_{\mathrm{D}}$ for this mixture is almost the same as that measured with the soap solution. The latter result evidences the fact that myristic acid is dominating the properties of the surfactant adsorption layers, despite being only a small fraction in the surfactant mixture. Note that the respective solution is clear (without precipitates), with bulk viscosity of $0.9 \mathrm{mPa} . \mathrm{s}$ and $\mathrm{pH}$ around 6.5, properties which are very similar to those of the original SLES + CAPB bulk solution without MAc.

The strong effect of the fatty acids on $E_{\mathrm{D}}$ is explained by their ability to pack very well in the adsorption layers, forming a surface condensed phase, ${ }^{75}$ which is characterized by high surface modulus and low tangential mobility of the surfaces. As explained in ref. 24 the increase in temperature leads to melting of the surface condensed phase, thus transforming the surfactant adsorption layer into a typical fluid layer with low surface modulus.

Summarizing, the role of the various components in the proposed surfactant mixtures is as follows: SLES and CAPB form mixed micelles with relatively high capacity for solubilisation of fatty acids. These micelles incorporate the acid molecules and supply them onto the solution surface. Due to the higher surface activity of the fatty acids, the latter are accumulated in the adsorption layer, despite their relatively low concentration in the bulk solution. Once a sufficiently high concentration of LAc and MAc molecules is reached on the surface, the acid molecules form surface condensed phase, thus sharply reducing the tangential surface mobility. A schematic presentation of the role of the various components and of the formation of surface condensed phase, dominated by fatty acids, is shown in Fig. 6.

Note that this general principle of designing surfactant mixtures, which beneficially combine the bulk and surface properties of their components, could be applied to a variety of surfactants with different headgroups and chain-lengths. Furthermore, if the surfactants are characterized with very strong specific interactions in the adsorption layers (e.g., having

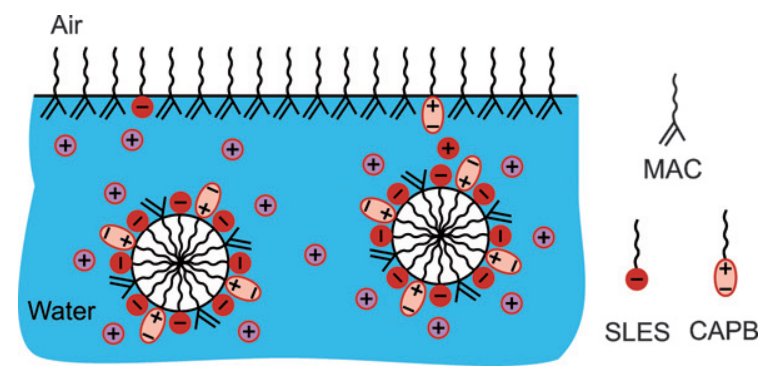

Fig. 6 A schematic presentation of the solubilisation of fatty acids in mixed surfactant micelles of CAPB and SLES, and of the formation of a surface condensed phase of fatty acids in the adsorption layer. ${ }^{24}$ This surface condensed phase is characterized by very high surface modulus, $E_{\mathrm{D}}>100 \mathrm{mN} / \mathrm{m}$, and renders low tangential mobility of the bubble surfaces. Upon increase of temperature above the phase transition temperature, the surface condensed phase melts and the adsorption layer transforms into a typical fluid layer with low surface modulus. oppositely charged headgroups), the properties of the surface adsorption layer could be governed by complexes of surfactant molecules, and thus could be very different from the surface properties, rendered by the individual components. These possibilities are demonstrated in the following section 3.2.

\subsection{Effect of cosurfactants on foam rheological properties}

As expected, the experiments showed that if the addition of cosurfactant leads to a significant increase in the surface modulus of a foaming solution, this cosurfactant switches the regimes of foam viscous friction. Illustrative experimental results are shown in Fig. 7 for foams stabilized by different SLES + CAPB + cosurfactant mixtures (at air volume fraction $\Phi=90 \%$ ). Plots of the dimensionless inside-foam viscous stress, $\tilde{\tau}_{V}(\mathrm{Ca})$, are shown in Fig. 7A, whereas the dimensionless foam-wall stress, $\tilde{\tau}_{W}\left(\mathrm{Ca}^{*}\right)$, is shown in Fig. 7B. As seen from these figures, all cosurfactants studied fall into two distinct groups, with respect to their effect on foam rheological properties:

(1) Cosurfactants which significantly increase the surface dilatational modulus of the SLES + CAPB solution. The
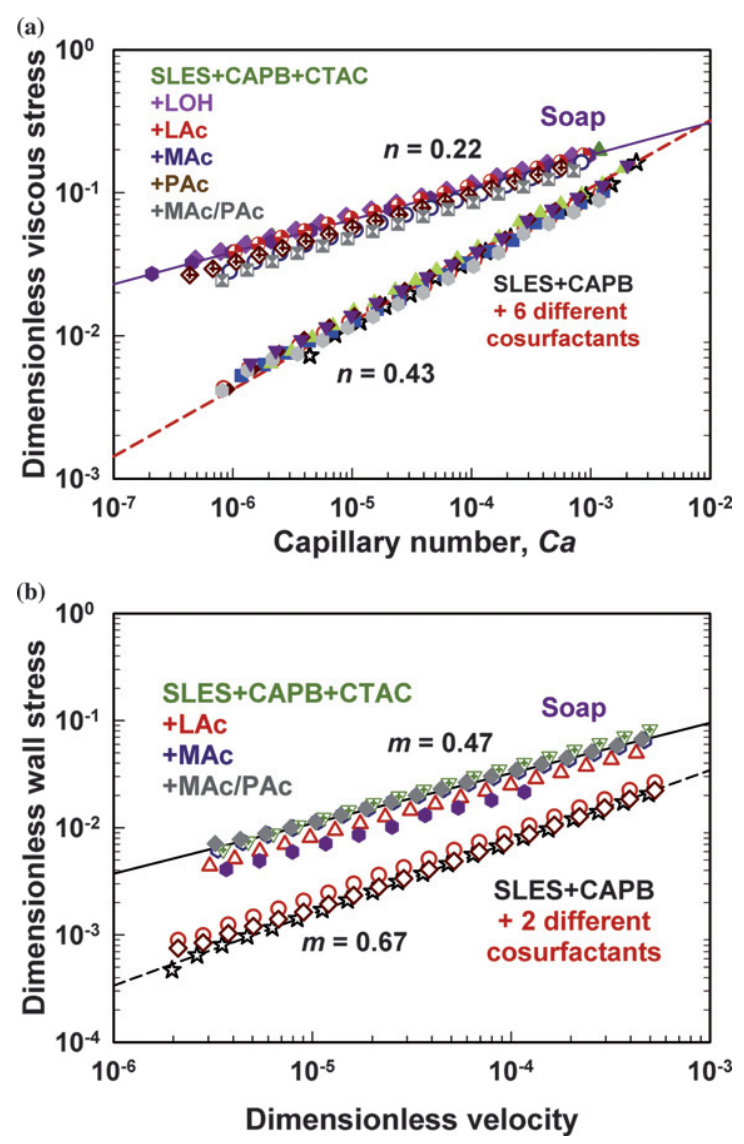

Fig. 7 (A) Dimensionless viscous stress, $\tilde{\tau}_{V}$, vs. capillary number, $C a$, and (B) Dimensionless wall stress, $\tilde{\tau}_{W}$, vs. dimensionless velocity, $\mathrm{Ca}^{*}$, for foams stabilized by SLES + CAPB mixture $(0.33+0.17 \mathrm{wt} \%)$, without and with different cosurfactants added $(0.02 \mathrm{wt} \%)$, which differ in their headgroups. Results obtained with soap solution (mixture of potassium salts of fatty acids, used at $\mathrm{pH}=10.2$ ) are also shown for comparison. The lines are drawn as eye-guides with the slope indexes $n$ and $m$, as indicated on the figures. 
experimental results for $\tilde{\tau}_{V}(\mathrm{Ca})$ and $\tilde{\tau}_{W}\left(\mathrm{Ca} a^{*}\right)$, obtained with these systems, are much higher than those obtained with foams formed from the original SLES + CAPB solution, and the respective power-law indexes are $n \approx 0.22 \pm 0.02$ and $m \approx 0.50 \pm 0.03$ (similar to those for soap solutions). Cosurfactants belonging to this non-trivial group are the fatty acids (LAc, MAc, PAc), lauryl alcohol ( $\mathrm{LaOH})$, and the cationic surfactant cetyltrimethylammonium chloride (CTAC).

(2) Cosurfactants which do not significantly change the surface rheological properties of the SLES + CAPB surfactant layer and, as a consequence, they affect neither the inside-foam friction nor foam-wall friction. For these cosurfactants, all experimental data $\tilde{\tau}_{V}(\mathrm{Ca})$ fall into a single master line with $n \approx 0.46 \pm 0.03$ and all data for $\tilde{\tau}_{W}$ vs. $C a^{*}$ merge onto a single line with index $m \approx 0.66$ \pm 0.03 . Wide variety of cosurfactants (anionic, non-ionic, and zwitterionic) was found to belong to this group.

Note that the cosurfactants tested have rather different headgroups and chain-lengths, which evidences that the quoted values of the flow indexes $n$ and $m$ are not chemistry-specific and, therefore, they reflect generic mechanisms of foam viscous friction.

\section{Theoretical explanation of the effects of surface mobility}

In the current section we briefly describe the main theoretical concepts, which explain the observed strong effect of bubble surface mobility on foam rheology. The theoretical models described in ref. 16,17,23 and 46 are used as basis for the following consideration. The relationship with some other recent theoretical explanations ${ }^{76-81}$ and experimental results ${ }^{25,48-50}$ is discussed in section 5 .

It is worthwhile noting in advance that the explanations of the effect of surfactant type on inside-foam friction and on foamwall friction are conceptually different. The theoretical analysis shows that HSM surfactants increase the foam-wall viscous stress (compared to LSM surfactants) mainly by increasing the area of the bubble-wall contact zone, in which the viscous friction is significant (due to immobilization of the bubble surface), ${ }^{23,46}$ whereas the higher inside-foam friction for these surfactants is due to an additional mechanism of energy dissipation in the surfactant adsorption layers on bubble surfaces. ${ }^{17}$ The ultimate reason for these different explanations is that the bubble-wall friction occurs at fixed shape of the bubbles (i.e. at constant surface area of the bubbles), whereas the bubble-bubble collisions inside sheared foam lead to perpetual variation of the bubble surface area around a mean value.

\subsection{Viscous friction inside sheared foam}

Detailed theoretical model for the viscous friction in homogeneously flowing foams and concentrated emulsions is described in ref. 16 and 17. In the current section we present only the main ideas of the model and the most important final results from the performed numerical calculations.

To develop a complete self-consistent model, we considered idealized sheared foam containing monodisperse bubbles, arranged in face-centred-cubic (fcc) structure. Homogeneous foam flow with shear rate, $\dot{\gamma}$, corresponds to motion of the
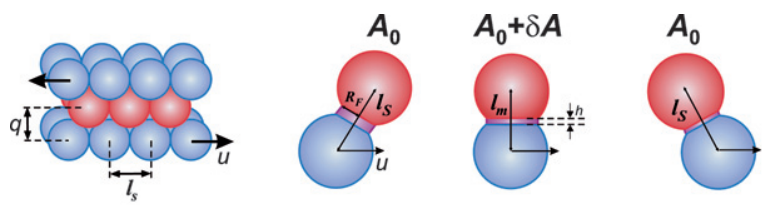

(A)
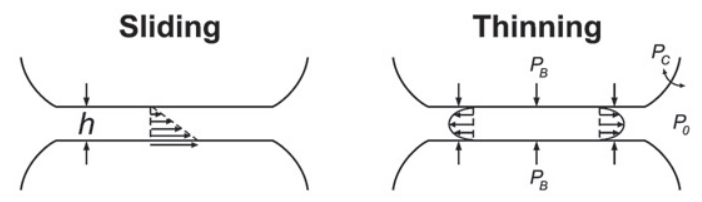

(B)
Fig. 8 (A) A schematic presentation of the processes of formation, thinning, and disappearance of a foam film between two neighbouring bubbles in sheared foam. ${ }^{16,17}$ Note that the process of formation and expansion, and the subsequent shrinking and disappearance of the foam film is accompanied with change in the total surface area of the colliding bubbles; (B) a schematic presentation of the two "elementary" processes determining the film dynamics in sheared foams - sliding of film surfaces (driven by the imposed foam flow) and film thinning (driven by the higher pressure in the bubbles, $P_{\mathrm{B}}$, as compared to the pressure in the liquid outside the film, $P_{0}$ ). The viscous dissipation is predominantly due to the sliding motion, while the film thickness (which determines the local shear rate of the liquid in the foam film and, hence, the rate of viscous dissipation of energy) is governed by the thinning process.

neighboring planes of bubbles with relative velocity, $u=q \dot{\gamma}$, where $q$ is the plane-to-plane distance, see Fig. 8A. To calculate the macroscopic foam viscous stress we used a balance of the work performed by the external stress, which drives the foam flow, and the energy dissipated inside the foam. This balance can be represented in the following convenient form: ${ }^{16}$

$$
\langle\dot{E}\rangle=\tau_{V} \dot{\gamma}
$$

where $\tau_{V}$ is the viscous stress and $\langle\dot{E}\rangle$ is the rate of energy dissipation per unit foam volume. Note that this relation is equivalent to the law of energy conservation and, therefore, it should be satisfied, whatever model is proposed to describe the foam flow.

Equation (4) converts the problem for calculating the foam viscous stress, $\tau_{V}$, into a problem of calculating $\langle\dot{E}\rangle$. The latter could be solved by considering the various possible mechanisms of viscous dissipation of energy, at the structural level of colliding bubbles. We found ${ }^{16,17}$ that the experimental data obtained with various foams and concentrated emulsions could be described by considering two major mechanisms of viscous friction: (1) in the foam films, formed between two colliding bubbles, and (2) in the surfactant adsorption layers on the bubble surfaces. The second contribution is important for the systems with high surface modulus only. These two contributions are considered separately below.

4.1.1. Friction inside film region. The viscous dissipation of energy in the foam films is due to the relative motion of the bubbles with respect to each other, which leads to the appearance of a local velocity gradient of the fluid confined in the film, see Fig. 8B (tangentially immobile bubble surfaces are assumed in the entire consideration). Note that no permanent foam films exist in the flowing foam - the foam films are formed when the 
distance between the centres of two colliding bubbles becomes smaller than the sum of bubble radii, ${ }^{11-13}$ and disappear when the bubbles are detached from each other, dragged by the flow, see Fig. 8A.

To calculate the velocity distribution in the foam film, and the resulting energy dissipation rate, we considered theoretically the dynamics of the liquid inside the foam film, by using lubrication approximation. This consideration showed ${ }^{17}$ that the liquid motion inside the film could be decomposed into two "elementary" processes, which occur simultaneously: (1) relative sliding of the film surfaces, which is due to the bubble motion, Fig. $8 \mathrm{~B}$, and (2) thinning of the foam film, which is due to the higher dynamic pressure inside the film (imposed by the capillary pressure of the bubbles, $P_{\mathrm{C}}$ ), as compared to the film exterior, see Fig. 8B. The numerical estimates showed that the main energy dissipation in the film is due to the sliding motion of the bubbles (viz. process 1), however, the film thinning (process 2) should be also considered explicitly, because it determines the film thickness and the resulting velocity gradient inside the film.

Following the standard hydrodynamic approach and using reasonable assumptions, in ref. 16 and 17 we calculated the instantaneous film thickness, $h(t)$, and film radius, $R_{\mathrm{F}}(t)$, in the process of bubble-bubble collision. Next, we calculated the rate of energy dissipation inside the foam film and the respective energy dissipated during the entire period of existence of one film. Finally, by using eqn (4) we derived formulas for calculating the viscous stress, in the form of explicit integrals which were solved numerically. The respective numerical results were described very well by the following interpolating formula: ${ }^{17}$

$$
\tilde{\tau}_{V F} \approx 1.16 C a^{0.47} \Phi^{5 / 6}(\Phi-0.74)^{0.1} /(1-\Phi)^{0.5}
$$

where $\tilde{\tau}_{V F}=\tau_{V F} R_{0} / \sigma$ is the dimensionless stress related to the friction in the foam films and $R_{0}$ is bubble radius. The subscript "VF" denotes viscous friction inside the films.

Note that eqn (5) should be used in its range of validity only. At very high volume fractions, $\Phi \rightarrow 1$, the films become very thin due to the high capillary pressure of the bubbles, so that the forces acting between the film surfaces (disjoining pressure), which were neglected in the derivation of eqn (5), become important. The upper limit of $\Phi$ can be estimated by comparing the thickness of the dynamic film, $h \approx C a^{1 / 2} R_{0} / 4$, with the range of surface forces $(c a .10 \mathrm{~nm}) .{ }^{17}$ The lower limit of $\Phi$ is set by the model assumption that the bubbles form planar films while sliding along each other. The comparison of the model predictions with various experimental data showed that eqn (5) is applicable at least in the range $0.80<\Phi<0.98 .{ }^{17}$

This model predicts $\tilde{\tau}_{V F} \propto C a^{1 / 2}$, i.e. $n \approx 1 / 2$, which is in very good agreement with many experimental results obtained with various foams and emulsions, ${ }^{1,9,17,23-25,54-55}$ see also Fig. 2, 3 and 7A. Note that the nice agreement between the predictions of eqn (5) and the experimental results is obtained without using any adjustable parameter, which is a very good indication that this model adequately describes the main mechanism of viscous dissipation in the foams and concentrated emulsions, stabilized by low-surface-modulus surfactants. The higher viscous stress, measured with foams stabilized by high-surface-modulus surfactants, shows that additional contributions in the viscous stress should be considered in these systems (see section 4.1 .2 below).
More sophisticated versions of this model were also developed in ref. 17 to account for the possible effects of (1) viscous dissipation of energy in the meniscus region surrounding the foam films, and (2) surface forces between the foam film surfaces. The numerical calculations showed that the energy dissipation in the meniscus region could be neglected in most cases and that the effect of surface forces becomes significant at low shear rates, when the thickness of the dynamic foam films becomes comparable to the range of surface forces. The latter effect is more important for emulsions and foams containing very small bubbles, due to the strong dependence of the rate of film thinning on the film diameter, see eqn (14) below. Most interestingly, the theoretical analysis of the effect of attractive surface forces (e.g., of van der Waals or depletion interactions) provided a direct mechanistic explanation of the observed "jamming" (liquid-tosolid transition) upon decrease of the shear rate, when the foam yield stress is reached. ${ }^{18}$

One of the important conclusions from the model developed in ref. 16 and 17 is that the appropriate mean bubble size for description of viscous friction in sheared polydisperse foams is the mean volume-surface radius, $R_{32}$. This conclusion directly emerges from the dimensional analysis of the theoretical expression for the viscous stress ${ }^{16,17}$

$$
\tau_{V F}=\langle\dot{E}\rangle / \dot{\gamma}=1.63 E_{F} \Phi / V_{B}
$$

Here $E_{\mathrm{F}}$ is the energy dissipated in one foam film from the moment of its formation to the moment of its disappearance and $V_{\mathrm{B}}$ is the bubble volume. Since $E_{\mathrm{F}}$ is proportional to $R^{2}$ (see Fig. 6 in ref. 17) and $V_{\mathrm{B}}$ is proportional to $R^{3}$, where $R$ is the characteristic bubble size, one sees that $\tau_{V F}$ should be scaled with $\left\langle R^{3}\right\rangle /\left\langle R^{2}\right\rangle=R_{32}$. Thus we justify the use of $R_{32}$ as a characteristic size for the viscous friction in polydisperse foams and emulsions, for the systems in which the viscous dissipation in the foam films is dominant.

4.1.2. Role of surface dissipation (surface viscous modulus). The analysis of bubble dynamics in sheared foam shows that the perpetual formation and disappearance of foam films between colliding bubbles leads to concomitant variation of the bubble surface area around its mean value, $A_{0}{ }^{1,6-9,16,17,51}$ To account for the viscous dissipation in the sheared foams, which is due to the surface dilatational viscosity, we approximated the consecutive expansions and contractions of the bubble surface in the flow with oscillatory deformation of amplitude, $a_{0}$, and estimated the contribution of the surface dissipation to the total dissipated energy in the foam. ${ }^{17}$ Here $a_{0} \equiv \delta A / A_{0}$ denotes the relative amplitude of the bubble area deformation, as a result of the film formation between two sliding bubbles, see Fig. 8A. By integrating the energy dissipation rate over one oscillatory cycle of bubble area expansion/contraction (due to the appearance and disappearance of one foam film) we obtained the following expression for the contribution of the surface dissipation, $\tau_{V S}$, into the total viscous stress: ${ }^{17}$

$$
\tilde{\tau}_{V S} \equiv \tau_{V S} R_{0} / \sigma \approx 9.8 \pi\left(E_{L D} / \sigma\right) \Phi a_{0}^{2}
$$

where $E_{\mathrm{LD}}$ is the surface dilatational loss modulus (viscous modulus). Note that the dependence of $\tau_{V S}$ on $\dot{\gamma}$ appears only 
through the possible dependences of $E_{\mathrm{LD}}$ and $a_{0}$ on $\dot{\gamma}$. Note also that the same theoretical approach could be directly applied to describe surface dissipation in foams and concentrated emulsions, subject to oscillatory deformation (e.g., of shear or extensional type).

The viscous stress in sheared foams, which are stabilized by surfactants with high surface loss modulus, $E_{\mathrm{LD}}$, contains contributions from both energy dissipation in foam films, eqn (5), and energy dissipation in adsorption layers, eqn (7), that is $\tau_{V}$ $=\tau_{V F}+\tau_{V S}$. No theoretical model is currently available to predict the dependence of $E_{\mathrm{LD}}\left(a_{0}\right)^{2}$ on $\dot{\gamma}$. Therefore, we used experimental data from the foam rheometry to estimate the product:

$$
\left(E_{L D} / \sigma\right) a_{0}^{2} \approx B \dot{\gamma}^{0.18}
$$

where $B \approx 1.26 \times 10^{-3}$ was determined for LAc containing foams and $B \approx 2.12 \times 10^{-3}$ for MAc-containing foams. ${ }^{17}$ The power index $n \approx 0.18 \pm 0.02$ in eqn (8), which expresses the dependence of $\tau_{V S}$ on $\dot{\gamma}$, was determined from the interpretation of the foam rheology data (after subtracting $\tau_{0}$ and $\tau_{V F}$ from the total shear stress) and still lacks a theoretical justification.

From eqn (5) and (7) one can estimate the ratio of the contributions of surface dissipation and inside-film dissipation of energy in sheared foams. Thus for typical foam with $\Phi=0.9$ one obtains:

$$
\frac{\tilde{\tau}_{V S}}{\tilde{\tau}_{V F}} \approx 9.9 B \frac{\sigma^{0.47}}{\mu^{0.47} R^{0.47} \dot{\gamma}^{0.29}}
$$

We see that the relative contribution of surface dissipation increases with the value of surface loss modulus (hidden in the value of $B$, which is roughly proportional to $E_{\mathrm{LD}} / \sigma$ ) and with the decrease of solution viscosity, mean bubble size, interfacial tension, and shear rate. As an example, for MAc-containing foams with $R_{32}=100 \mu \mathrm{m}, \sigma=22 \mathrm{mN} / \mathrm{m}$ and $\mu=1 \mathrm{mPa} . \mathrm{s}$, we estimate $\tilde{\tau}_{V S} / \tilde{\tau}_{V F} \approx 1.8$ at $\dot{\gamma}=100 \mathrm{~s}^{-1}$, whereas for more viscous solution, $\mu=10 \mathrm{mPa}$.s, this ratio becomes 0.6 , i.e. the inside-film dissipation becomes prevailing.

\subsection{Foam-wall viscous friction}

Detailed theoretical models for bubble-wall and foam-wall friction are presented in ref. $23,46,60-65,67$. Here we outline the main assumptions in these models and their final conclusions, with the major aim to explain the origin of the different powerlaw indexes observed experimentally.

All theoretical models of the viscous friction between bubble and smooth solid wall are based on calculations of the fluid velocity profile in the wetting film, formed in the bubble-wall contact zone, see Fig. 9. For these calculations, a "non-slip" boundary condition is used for the liquid flow at the solid wall, whereas different boundary conditions could be assumed for the bubble surface. For bubbles with tangentially mobile surfaces one assumes the so-called "stress-free" boundary condition which results in non-zero velocity of the fluid at the bubble surface (Fig. 9A), whereas for bubbles with tangentially immobile surfaces the non-slip boundary condition is appropriate (Fig. 9B). Once the velocity profile in the wetting film is
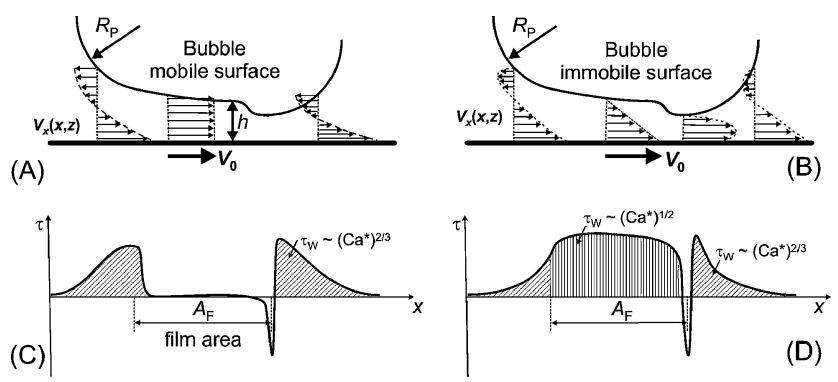

Fig. 9 A schematic presentation of the zone of bubble-wall contact with the profile of the fluid velocity for (A) tangentially mobile and (B) tangentially immobile bubble surfaces. ${ }^{46}$ (C,D) From the velocity profiles one can calculate the local viscous stress on the solid wall, $\tau(x)$, which can be integrated along the contact zone to calculate the total bubble-wall friction force, $F_{\mathrm{FR}}$. (C) In the case of tangentially mobile bubble surface, $\tau(x) \approx 0$ in the film region, so that the bubble-wall friction originates in the meniscus zones around the film only. (D) In the case of tangentially immobile bubble surface, the friction occurs in both zones of the film and in the surrounding meniscus regions. The theoretical models show that the friction in the meniscus regions scales as $\left(\mathrm{Ca}^{*}\right)^{2 / 3}$ for both mobile and immobile bubble surfaces, whereas the friction in the film region scales as $\left(\mathrm{Ca}^{*}\right)^{1 / 2}$ (for immobile surface). ${ }^{46,60,67}$

calculated, one can calculate and integrate the local viscous stress acting on the solid wall, $\tau(x)$ (see Fig. 9C,D), thus determining the total bubble-wall friction force, $F_{\mathrm{FR}},{ }^{60}$ and the average bubble-wall stress, $\tau_{W .}{ }^{23,46}$ In most theoretical models, the numerical calculations are made for idealized, infinitely-long cylindrical bubbles (2D-bubbles) for which $F_{\mathrm{FR}}$ is the force per unit length of the bubble-wall contact line. ${ }^{23,46,60-63}$ In ref. 23 and 46 it is shown that the results for such model 2D-bubbles could be applied to calculate $\tau_{W}$ for real 3D bubbles in foam contacting a solid wall, by averaging the viscous stress over the bubble-wall contact area.

The theoretical models predict that $\tau_{W} \propto\left(\mathrm{Ca}^{*}\right)^{1 / 2}$ for bubbles, in which the bubble-wall friction is dominated by the viscous stress inside the area of the wetting film, which is possible only if the bubble surface is tangentially immobile. ${ }^{23,46} \mathrm{In}$ contrast, $\tau_{W} \propto$ $\left(\mathrm{Ca}^{*}\right)^{2 / 3}$ is predicted for the systems, in which the bubble-wall friction is dominated by the viscous stress in the curved menisci regions surrounding the wetting film (viz. in the thinnest part of the Plateau border around the film). ${ }^{60,61}$

Let us explain more explicitly how these theoretical models explain the origin of the scaling laws observed experimentally. In the systems with mobile bubble surfaces, the local shear rate (gradient of liquid velocity) is almost zero inside the wetting film, while it is significant in the curved meniscus zones around the film, because the mass conservation law requires some liquid to be squeezed out of the converging entrance zone of the film (in which the liquid layer thickness decreases along the liquid flow) and some liquid to be sucked into the exit zone (in which the liquid layer thickness increases) ${ }^{60}$ see Fig. 9A. The flow in these two zones was analyzed in detail by Bretherton, ${ }^{60}$ who showed that the thickness of the formed wetting film, $h$, and the effective area of viscous friction, $A_{\mathrm{FR}}$ (i.e., the area in which the viscous stress is significant) scale as:

$$
h / R_{P}=B_{1}\left(C a^{*}\right)^{2 / 3}
$$




$$
A_{\mathrm{FR}} / R_{\mathrm{P}}{ }^{2}=B_{2}\left(C a^{*}\right)^{1 / 3}
$$

where $R_{\mathrm{P}}$ is radius of curvature of the meniscus around the wetting film and the numerical constants $B_{1}$ and $B_{2}$ are different for tangentially mobile and tangentially immobile bubble surfaces. ${ }^{60}$ Using these scaling laws, one can obtain for the mobile bubble surface the following explicit expressions for the bubble-wall friction force, $F_{\mathrm{FR}}{ }^{60}$ and the respective foam-wall viscous stress, $\tau_{W}{ }^{23}$

$$
F_{F R}=\mu V_{0} A_{F R} / h \approx 4.7\left(C a^{*}\right)^{2 / 3} \sigma
$$

$2 D$-bubble force per unit length of bubble-wall contact line mobile bubble surface

$$
\tau_{W} \approx 11.7\left(\frac{\sigma}{R_{32}}\right) f_{3}^{1 / 2}(\Phi)\left(C a^{*}\right)^{2 / 3}
$$

foam mobile bubble surface

where $\mathrm{f}_{3}(\Phi) \approx 1-3.2(\Phi /(1-\Phi)+7.7)^{-1 / 2}$ is a function, which depends on the air volume fraction only. ${ }^{1,23,46}$

In Bretherton model, ${ }^{60}$ it is explicitly assumed that the central zone of the wetting film moves with the velocity of the wall (in a plug flow) and, hence, there is no velocity gradient and viscous dissipation inside the film, ${ }^{60,67}$ see Fig. 9A and 9C. Note, however, that this assumption is inapplicable to the systems with strictly immobile bubble surfaces, because the bubble and the solid wall move with relative velocity $V_{0}$, thus creating velocity gradients in the central zone of the wetting film, Fig. 9B. ${ }^{23,46}$ The theoretical analysis showed ${ }^{46}$ that the friction force in this case contains two components, one coming from the central zone of the film, which scales with $\left(\mathrm{Ca}^{*}\right)^{1 / 2}$, and another component coming from the curved surfaces around the film, which scales with $\left(\mathrm{Ca}^{*}\right)^{2 / 3}$ :

$$
F_{F R} \approx 2.50 \sigma\left(C a^{*}\right)^{1 / 2}\left(\frac{R_{F}}{R_{P}}\right)^{1 / 2}+3.3 \sigma\left(C a^{*}\right)^{2 / 3}
$$

$2 D$-bubble force per unit length of bubble-wall contact line immobile bubble surface

$$
\tau_{W}=\frac{\sigma}{R_{0}}\left(1.25 C_{I F}\left(C a^{*}\right)^{1 / 2} \frac{f_{3}^{1 / 4}}{\sqrt{1-f_{3}^{1 / 2}}}+2.1 C_{I L}\left(C a^{*}\right)^{2 / 3}\right) f_{3}^{1 / 2}
$$

foam immobile bubble surface

where $C_{\mathrm{IF}}$ and $C_{\mathrm{IL}}$ are numerical constants. Subscript "I" is a reminder that these expressions are for immobile bubble surface, whereas subscripts "F" and "L" stand for the friction inside and outside the film area, respectively. The values $C_{\mathrm{IF}} \approx$ 3.7 and $C_{\mathrm{IL}} \approx 3.5$ were determined by comparing the predictions of eqn (13b) to rheological data ${ }^{25,46}$ (see comments in section 5.1).

The first term in eqn (13a) is proportional to the film area, whereas the second term is a weak function of the film size. Numerical calculations and experimental results showed ${ }^{46}$ that the first term dominates and the total friction force scales as $\left(\mathrm{Ca}^{*}\right)^{1 / 2}$, when the film radius is equal or larger than the radius of curvature of the Plateau border, $R_{\mathrm{F}} \geq R_{\mathrm{P}}$. In contrast, when $R_{\mathrm{F}}<R_{\mathrm{P}}$, the two terms are comparable, so that the effective power-law index is intermediate, $1 / 2 \leq m \leq 2 / 3$, and depends on the ratio $R_{\mathrm{F}} / R_{\mathrm{PB}}$.
In conclusion, the main effect of surfactants in the case of foam-wall friction is to change the surface mobility of the bubble surface. The bubbles with a high surface modulus behave as tangentially immobile and the power-law index is $m \approx 1 / 2$ (at sufficiently large film radius), whereas the bubbles with a low surface modulus behave as tangentially mobile and the powerlaw index is $m \approx 2 / 3$ (for arbitrary film radius), see Table 1 .

All consideration up to here was made under the assumption that the surfaces are either completely mobile (stress-free) or completely immobile, and that the liquid inside the wetting film is Newtonian (viz. its viscosity does not depend on shear rate). The reality could be more complex-for example, the surfaces could be partially mobile and, in this case, more complex boundary conditions at the bubble surface are required. ${ }^{11-13,63}$ To find a general solution for this theoretical problem is an extremely difficult task which has not been solved so far. However, theoretical models for some specific cases (diffusion or barrier control of surfactant adsorption), ${ }^{82-84}$ as well as some preliminary estimates accounting for the effects of surface elasticity ${ }^{85}$ have shown that the power-law index $m$ could vary in wide range, including values which are significantly lower than $1 / 2$. Similarly, if the liquid in the wetting film is shear thinning (its viscosity decreases with the increase of shear rate) one should expect a lower value of $m$, as compared to the case of a Newtonian liquid, with all remaining factors being the same - these results are summarized in the last row of Table 1.

It should be mentioned that the surfactants which give mobile bubble surfaces in the foam-wall experiments (SLES, CAPB, SDS) were shown to give immobile bubble surfaces in the insidefoam friction experiments. ${ }^{17,23,24}$ Most probably, the reason for this non-trivial result is the qualitatively different dynamics of the bubbles and of the respective thin films in the two processes: in the foam-wall friction experiments, the bubbles have a stationary shape and the films have constant radius and thickness (at given velocity of the wall). In contrast, the foam films between colliding bubbles in sheared foams have limited lifetime, and continuously change their thickness and radius, see Fig. 4 in ref. 17. Therefore, the viscous stresses exerted on the film surfaces and the mass-transfer of surfactant toward/from the bubble surface are qualitatively different in the two types of experiments, which may lead to different surface rigidity of the bubbles.

\section{Comparison with results obtained by other research groups}

In this section we compare our results to those obtained by several other research groups and discuss the possible mechanisms of viscous dissipation in foams. The focus is on steadily sheared, homogeneously flowing foams, but some other related systems (such as concentrated emulsions, dispersions of microgel particles, semi-concentrated foams and emulsions with $0.50<\Phi$ $<0.74)$ are also discussed.

\subsection{Steadily sheared 3D-foams}

Systematic series of rheological experiments with steadily sheared foams were performed recently by Marze et al. ${ }^{25}$ in which several foam stabilizers were compared-SDS, casein, and 
potassium cocoylglycinate GCK (amino-acid-based surfactant resembling the fatty acids in some aspects). Air volume fraction, $\Phi$, was varied in relatively wide range to clarify its effect on foam properties. Several important results were obtained in this study:

First, it was confirmed (by using cone-plate rheometry) that the power-law indexes for foams stabilized by SDS (typical LSM surfactant) are $n \approx 0.42$ and $m \approx 0.65$, and do not depend on $\Phi$. These results are in a very good quantitative agreement with our experimental results obtained in parallel plate geometry with foams and emulsions, stabilized by different surfactants, ${ }^{17,23,24}$ as well as with the results obtained by Princen and Kiss ${ }^{1,9}$ with concentrated emulsions in Couette geometry.

This quantitative agreement between experimental results obtained by three different groups, ${ }^{9,17,25}$ with such wide range of low-surface-modulus (LSM) systems, is a very clear indication that all these systems share the same generic mechanism of viscous friction. Furthermore, the fact that the theoretical model developed in ref. 16 and 17 can describe these systems without any adjustable parameter, means that the inside-foam friction is controlled by the viscous dissipation in the foam films (as assumed in the model). The foam-wall friction for these systems is also well understood in the frame of the adapted Bretherton analysis, ${ }^{60}$ with the only technical difficulty being that the model is developed for large 2D-foam films (like those formed when single long bubble is travelling along a capillary tube), whereas circular 3D-foam films are formed in the contact zone of the foam with solid wall. As shown in ref. 23 and 46, the Bretherton approach could be adapted to 3D-foam films by introducing an additional numerical constant, which was determined experimentally from the measured dependence $\tau_{W}\left(\mathrm{Ca}^{*}\right)$ - see the respective discussions in ref. 25 and 46.

The results for the inside-foam friction obtained with $\mathrm{GCK}^{25}$ were very similar to those obtained with SDS, i.e. GCK behaved as a typical LSM surfactant with respect to viscous dissipation inside sheared foam. In contrast, more complex behavior was found with GCK for the foam-wall friction-the index $m$ increased from 0.28 to 0.66 with the decrease of $\Phi$ from 0.95 to 0.85 . Based on this result, and assuming the GCK makes the bubble surfaces tangentially immobile, Marze et al. ${ }^{25}$ determined the values of the numerical constants $C_{\mathrm{IF}}$ and $C_{\mathrm{IL}}$ for $3 \mathrm{D}$-foam films with tangentially immobile surfaces, see eqn (13b). Although this analysis is interesting and worthy to be pursued, the obtained values should be verified with other surfactants in future experiments. The reason is that the obtained similar results for SDS and GCK (for inside-foam friction) indicate that GCK has no very high surface modulus (data for this modulus are not presented in the original paper ${ }^{25}$ ), thus leaving open the possibility that the observed variation of $m$ with GCK-stabilized foams could be related to a partial mobility of the bubble surface in the foam-wall experiments.

The results obtained with casein were very different from those obtained with SDS and GCK (and different from those described in sections 2 and 3 above). Unfortunately, a quantitative analysis of these results is impossible at the present moment because, as explained by the authors, ${ }^{25}$ the films stabilized by casein are very thick and inhomogeneous, due to the presence of adsorbed protein aggregates on the air-water interface. The latter circumstance means that (1) the effective viscosity of the material inside the films could be rather different from the viscosity of the original foaming solution, viz. the scalings involving solution viscosity are not relevant, and (2) the film thickness is not determined by the dynamic pressure in the films (as it is the case with the other surfactants and as assumed in most theoretical models), but by steric repulsion created by the protein aggregates. $^{25}$

In another recent study, Soller and Koehler ${ }^{48}$ designed an original experimental setup with Couette geometry (called the "foam drainage rheology technique"), which allows one to measure the rheological properties of draining foams, by continuous perfusion of surfactant solution on top of the foam. Commercial surfactant was used as the foam stabilizer (Dawn dish-washing liquid, product of Procter \& Gamble), which provides tangentially mobile bubble surfaces, see section 5.3 below. Experimental results for wide range of air volume fractions were presented, which were qualitatively different from those reported in ref. 9, 17, 23-25, 54 and 55. For example, the data were not described by the Herschel-Bulkley model and another semi-empirical equation was proposed to account for the dependence of the viscous stress on $C a$ and $\Phi$. The most probable reason for the observed differences between the results of Soller and Koehler ${ }^{48}$ and those of the other authors is the interesting observation $^{86}$ that the thickness of the foam films in draining foams could be strongly affected by the process of liquid drainage, via a mechanism which is still poorly understood. Because the friction between bubbles in sheared foam is directly related to the film thickness, ${ }^{17}$ any influence of liquid drainage on film thickness will directly affect the measured viscous stress. Therefore, one should not expect agreement of the experimental results obtained in ref. 48 with the results obtained by other authors with non-draining foams and emulsions. Nevertheless, the experimental setup and the results obtained in ref. 48 deserve special attention, because the rheological properties of draining foams could be of particular interest in various practical applications.

In two subsequent papers, Pilon and co-workers ${ }^{49,50}$ described the rheological properties of sheared foams containing small bubbles $\left(R_{32}\right.$ in the range $\left.30-50 \mu \mathrm{m}\right)$ with an intermediate volume fraction ( $\Phi$ varied between 0.54 and 0.70 ), stabilized by three different surfactants-nonionic, cationic, and anionic of different concentrations. Pipe-flow rheometry was used and the power-law index $n$ was found to fall in the narrow range between 0.60 and 0.66 for all systems studied. The authors interpreted these data as a dependence $\tau_{V} \propto \mathrm{Ca}^{2 / 3}$ (i.e. $n=2 / 3$ ), which was assumed to be a result of predominant friction in curved meniscus regions. ${ }^{50}$ Indeed, such a possibility is provided by the theoretical model presented in ref. 17 (see the second term in eqn (54) and the related discussion in ref. 17), which is, however, developed for concentrated systems with $\Phi>0.74$. Therefore, a firm theoretical explanation of the experimental results presented in ref. 40 and 41 and, more generally, of the viscous friction in foams with intermediate bubble volume fraction, is still missing. One interesting observation in ref. 49 and 50 was that the viscous friction depended strongly on bubble polydispersity (probably because $\Phi$ was around the sphere closepacking value, $\Phi_{\mathrm{CP}}$ ), whereas the results obtained at higher $\Phi \geq$ 0.80 do not indicate such strong dependence., ${ }^{9,17,23}$

Wide variety of experimental results was obtained with emulsions of intermediate and high drop volume 
fraction. ${ }^{1,9,52,54,55,57,58,87-89}$ However, the results obtained by the various groups differ significantly in the values of the flow-index $n$ (typically measured to be between 0.5 and 1 ), so that it seems impossible at the present moment to sort out these results into characteristic groups, like those discussed above for foams (except for the emulsions with $n \approx 1 / 2$, see Fig. 2). Note that due to the small drop size and the related faster thinning of the emulsion films (see eqn (14) below), the emulsions are affected much more than foams by the possible effects of the surface forces acting in the films, which makes very difficult the quantitative analysis of rheological data. Therefore, we will not discuss here the results obtained with such emulsions.

Interestingly, several results obtained with soft microgel particles $^{90-92}$ resemble qualitatively the results obtained with foams of tangentially immobile surfaces. For example, the microgel-wall friction was found to scale with $m=1 / 2$, and the inside-gel viscous friction was found to scale with $n=1 / 2$. Thus interesting analogies between foams and microgel dispersions have been noticed, ${ }^{25,91,92}$ which deserve further quantitative investigation.

\subsection{Steadily sheared $2 D$-foams, the role of foam polydispersity, and formation of equilibrium foam films in sheared foams}

Recently, 2D-foams (composed of monolayers of bubbles) have attracted a considerable amount of attention from several research groups, mainly because of the possibility for direct observation of the detailed bubble dynamics in such flowing foams. ${ }^{76-81,93-100}$ These experiments proved to be very valuable in analyzing several important phenomena (e.g., those related to non-homogeneous flows) and to clarify some common features of the different types of "yield stress systems", such as soft glassy materials, granular materials, foams, and emulsions. Also, the theoretical modelling of 2D-foams is technically much simpler, as compared to the $3 \mathrm{D}$-foams, so that the theoretical concepts could be more easily tested with $2 \mathrm{D}$-foams.

On the other hand, a deeper analysis of the results from these experiments has shown clearly that 2D-foams have specific features, which do not allow a direct transfer of the main conclusions to 3D-foams. For example, the values of the flow indexes and the character of shear-localization phenomena were shown to be affected strongly by the covering solid wall (present in most of the experiments with 2D-foams) which creates additional bubble-wall friction force without direct analog in 3D-foams. ${ }^{76-80}$ Even when the solid wall is absent, the measured flow indexes are often different from those measured with 3D-foams of the same surfactants (for example, $c f$. the results with Dawn presented in section 5.3 below to those presented in ref. 79-81), which evidences for differences in the bubble dynamics for these two types of systems.

In recent studies with $2 \mathrm{D}$-foams, ${ }^{76-80}$ a conceptually different explanation of the power-law index $n \approx 1 / 2$ was proposed. Based on a previous model by Durian, ${ }^{101,102}$ several research groups performed numerical simulations of the viscous friction in 2Dfoams, assuming that the thickness of the foam films between neighboring bubbles does not depend on the shear rate. ${ }^{76-80}$ From the viewpoint of foam film dynamics, this is possible only if the film thickness in the sheared foams is equal to the equilibrium film thickness i.e. the time for film thinning to the equilibrium film thickness should be shorter than the contact time of the bubbles in sheared foam. Such an assumption would immediately lead to power-law index $n \approx 1$, if a regular bubble flow (almost straight bubble trajectories) and tangentially immobile bubble surfaces are assumed, ${ }^{17}$ like in the model illustrated in Fig. 8. However, the numerical simulations showed that for polydisperse foams, the bubble flow could be irregular (at the low shear rates that were modelled) and, as a result, a power-law index $n \approx 1 / 2$ for the macroscopic foam flow was calculated. ${ }^{76-78}$ Furthermore, such an index was measured experimentally with slowly sheared 2D-foams, in which the bubbles were observed to follow irregular, curved trajectories. ${ }^{79,81}$ On this basis, a hypothesis was put forward that these results should be representative for the typical polydisperse foams. ${ }^{\mathbf{7 6 - 7 8 , 8 1}}$

To clarify the role of bubble polydispersity in foam viscous friction, we performed additional experiments with monodisperse 3D-foams. In Fig. 10 we compare the measured viscous stress for monodisperse and polydisperse 3D-foams, which both show $n \approx 1 / 2$. Results for different gaps between the parallel plates, corresponding to different number of bubble layers in the sheared monodisperse foams, are plotted to prove that the result is independent of the thickness of the foam sample. Optical observations showed that the bubbles were regularly flowing in both types of samples (except for the lowest shear rates, $\dot{\gamma}<0.2$ $\mathrm{s}^{-1}$ ). Thus we can conclude from these experiments that the effect of bubble polydispersity is certainly not decisive for the measured in our experiments power-law index, $n \approx 1 / 2$.

The comparison of the experimental conditions in the experiments performed with 2D-foams ${ }^{79-81}$ and $3 \mathrm{D}$-foams ${ }^{17,23,24}$ showed that the main difference seems to be the range of shear rates studied - it is much lower in the experiments with 2D-foams (typically $<0.1 \mathrm{~s}^{-1}$ ) and is thus, close to the critical shear rates for foam jamming (liquid-to-solid transition), at which the bubbles attach to each other and the foam flow non-homogeneously. In contrast, the experiments aimed at measuring the viscous stress in steadily sheared 3D-foams are typically made at relatively high shear rates (between 0.2 and $200 \mathrm{~s}^{-1}$ ), which are well above the jamming transition, viz. in the regime of well developed, regular foam flow.

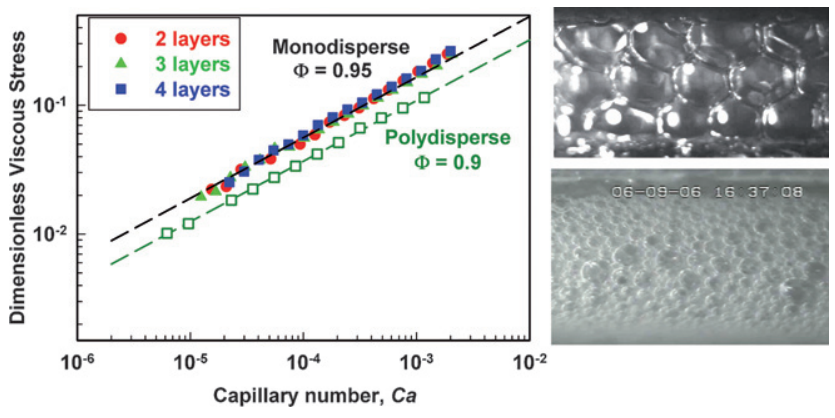

Fig. 10 Comparison of the dimensionless viscous stress, measured with monodisperse and polydisperse foams, stabilized by SLES + CAPB surfactant mixture. For the monodisperse foam, the data measured at different gaps between the parallel plates (corresponding to different numbers of the sheared bubble layers) are shown to demonstrate that the result is independent of gap width. The lines represent the theoretical predictions, eqn (5). The images show the cases of monodisperse foam with three layers (top) and polydisperse foam (bottom) as observed between the parallel plates. 
Therefore, we suppose that the theoretical models predicting $n \approx$ $1 / 2$ at constant film thickness ${ }^{76-81}$ could be representative only for foams and emulsions sheared at low rates, close to the jamming transitions, when the flow of polydisperse foams is non-homogeneous. At high shear rates, dynamic foam and emulsion films are formed between the neighboring bubbles and drops - the thickness of these films must depend on the applied shear rates and, therefore, one of the main assumptions of the theoretical models in ref. 76-81 is not justified. Also, direct optical observations show that the 3Dfoam flow at high shear rates is rather homogeneous and regular, independently of foam polydispersity. ${ }^{18,21}$

The above conclusion could be presented in quantitative terms, by estimating the time for film thinning down to the equilibrium film thickness: ${ }^{10,11,15}$

$$
t_{D R} \approx \frac{3}{4} \frac{\mu R_{F}^{2}}{P_{C} h_{E Q}^{2}} \approx \frac{3}{8} \frac{\mu R_{0} R_{F}^{2}}{\sigma h_{E Q}^{2}}
$$

which is obtained after integrating Reynolds equation for the rate of film thinning. Here $h_{\mathrm{EQ}}$ is the equilibrium film thickness, $R_{\mathrm{F}}$ is film radius, and $P_{\mathrm{C}} \approx 2 \sigma / R_{0}$ is capillary pressure of the bubbles. Taking for approximate estimate $h_{\mathrm{EQ}}=10 \mathrm{~nm}, \sigma=30$ $\mathrm{mN} / \mathrm{m}$, bubble radius $R_{0}=100 \mu \mathrm{m}, R_{\mathrm{F}}=R_{0} / 2$, and $\mu=1 \mathrm{mPa} . \mathrm{s}$, one finds that the characteristic time for film drainage is $t_{\mathrm{DR}} \approx$ $30 \mathrm{~s}$. Taking into account the fact that the contact time of two neighboring bubbles in sheared foams is of the order of $1 / \dot{\gamma}$, we see that thinning of the film to its equilibrium thickness is possible only in the case of very low shear rates in the foams, $\dot{\gamma}<$ $0.1 \mathrm{~s}^{-1}$. Note that eqn (14) is not quantitatively accurate for large, millimeter sized bubbles, for which additional effects due to gravity and non-homogeneous film thickness ${ }^{103,104}$ (both leading to accelerated film thinning) become significant. Note also that, due to the strong dependence on $R_{0}$ and $R_{\mathrm{F}}$, the characteristic time for film drainage is much shorter for micrometer and submicrometer emulsion droplets, so that equilibrium films could be formed at much higher shear rates in emulsions.

\subsection{Properties of some commercial surfactants, used in foam studies}

It is rather common in foam studies to use commercial surfactants, because the latter are optimized to give very stable foams. Most widely used are Gillette shaving foams,56,105,106 and foams produced from Dawn dish-washing liquid (product of Procter \& Gamble). ${ }^{38,39,79,80}$ From the viewpoint of the current study, it is of significant interest to classify these commercial surfactants with respect to the rheological properties of the respective foams. With this aim, we measured the inside-foam and foam-wall friction for such foams and the obtained results are compared in Fig. 11 with results, representative for tangentially mobile and tangentially immobile bubble surfaces.

One sees from Fig. 11, that the results obtained with Dawn practically coincide with those for typical surfactants with mobile surfaces. ${ }^{23,24}$ Furthermore, we measured the surface modulus of this system to be very low, $E_{\mathrm{D}}<1 \mathrm{mN} / \mathrm{m}$. All these results confirm the existing view that Dawn surfactants render tangentially mobile bubble surfaces. ${ }^{38,39}$

In contrast, the results obtained with Gillette shaving foam are very close to those for immobile bubble surfaces, thus placing this system in the category of high-surface modulus (HSM)
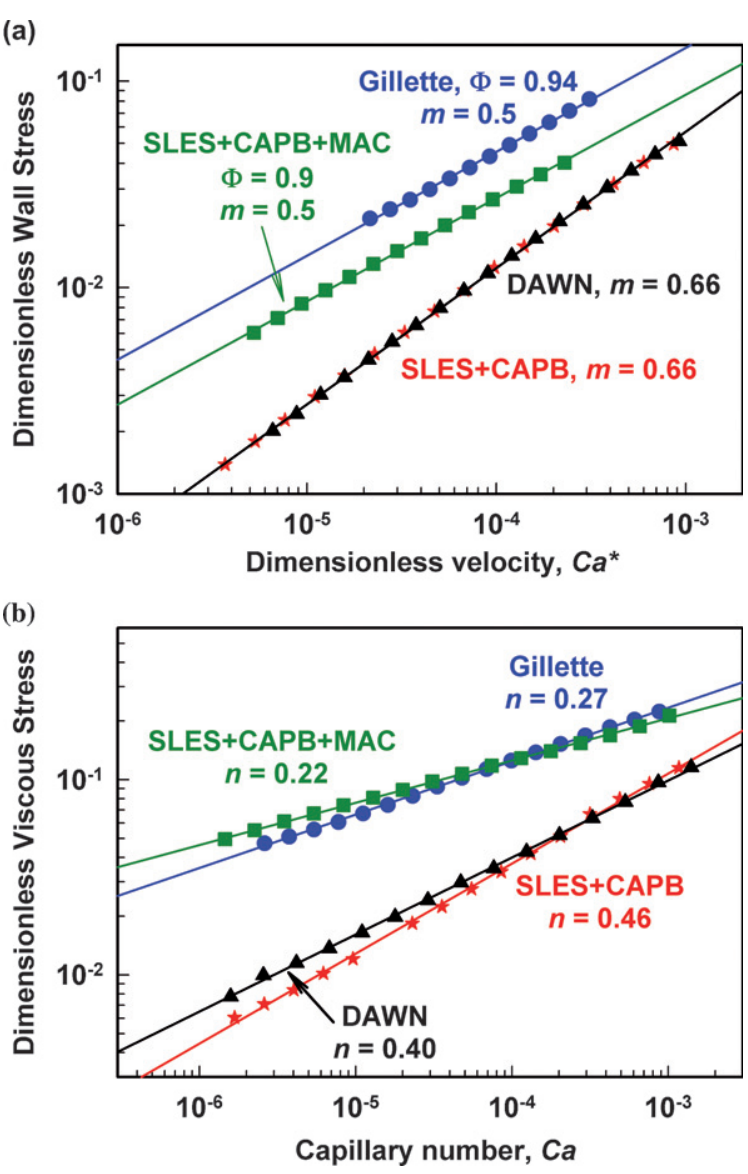

Fig. 11 Comparison of the dimensionless viscous stress for (A) foamwall friction, and (B) inside-foam friction, measured with Gillette foam and with foams generated from solutions of Dawn, SLES + CAPB, and SLES + CAPB + MAc. From the measured foam-wall stress, the unknown viscosity of the solution in Gillette foam was determined, $\mu \approx 5$ $\mathrm{mPa} . \mathrm{s}$, and used to plot the data in dimensionless form. The lines are drawn as eye-guides with the slope indexes $n$ and $m$, as indicated on the figures.

foams. Note that according to its producer (Gillette UK Ltd), the formulation used in our experiments contains palmytic and stearic acids, so that one could expect its behaviour to be closer to our fatty acid-containing surfactant mixtures, described in section 3 .

Thus we conclude that Dawn-stabilized foams are typical examples for foams with tangentially mobile bubble surfaces, whereas Gillette foams are typical examples for foams with tangentially immobile bubble surfaces. Note, however, that the viscosity of the foaming solution in different Gillette samples could vary, because these are commercial products with complex composition (changed on regular basis to improve product performance in customer use), which makes Gillette foams not very suitable for quantitative studies of foam dynamics.

\section{The role of surfactant type in bubble breakup in sheared foams}

Bubbles in intensively sheared foams could break under the effect of viscous stresses, created as a result of friction with 
neighbouring bubbles. This breakup process, which often determines the bubbles size distribution in sheared foams, is still poorly understood. In this section we discuss briefly recent experimental results, ${ }^{21}$ which illustrate the important effect of the dynamic surface properties of foaming solution on the process of bubble breakup.

To quantify and analyze the effect of surfactant type on the mean size and polydispersity of the bubbles formed during foaming, we performed systematic experiments, in which a foam containing initially large bubbles was subject to a given shear rate or given shear stress, sufficiently high to induce bubble breakup into smaller bubbles. ${ }^{21}$ These experiments showed that the bubbles formed after foam shearing are much smaller when highsurface modulus (HSM) surfactants are used as foam stabilizers, in comparison with the foams stabilized by LSM surfactants, under otherwise equivalent conditions. This trend is illustrated in Fig. 12, where the results from the following experimental protocol are shown: initial foams containing large bubbles with $R_{32} \approx 500 \mu \mathrm{m}$ and $R_{\mathrm{V} 95}=800 \mu \mathrm{m}$ are sheared for $3 \mathrm{~min}$ at a shear rate of $150 \mathrm{~s}^{-1}$. Here $R_{\mathrm{V} 95}$ is the radius of those bubbles, for which 95 vol. $\%$ of the dispersed air is confined in bubbles with smaller radius (this is a standard experimental measure for the size of the biggest drops in emulsions ${ }^{107-109}$ and here we adapt it for characterization of the biggest bubbles in foams). The foams are stabilized by the two types of surfactant mixtures discussed in section 3 and have the same air volume fraction, $\Phi=0.95$. The fact that the measured stress remains constant for the SLES + CAPB stabilized foam (see Fig. 12), shows that the bubbles in this foam do not break under these conditions - indeed, the bubble size distributions measured before and after shearing coincided for this foam. In contrast, at the same shear rate, the measured stress significantly increased, while the bubble size decreased during shearing the foam stabilized by SLES + CAPB + MAc, Fig. 12.

The analysis of the experimental results from a large set of systems revealed an ultimate relation between the effects of surfactants on foam rheological properties and on bubble breakup ${ }^{21}$ - bubbles with radius $R$ would break in sheared

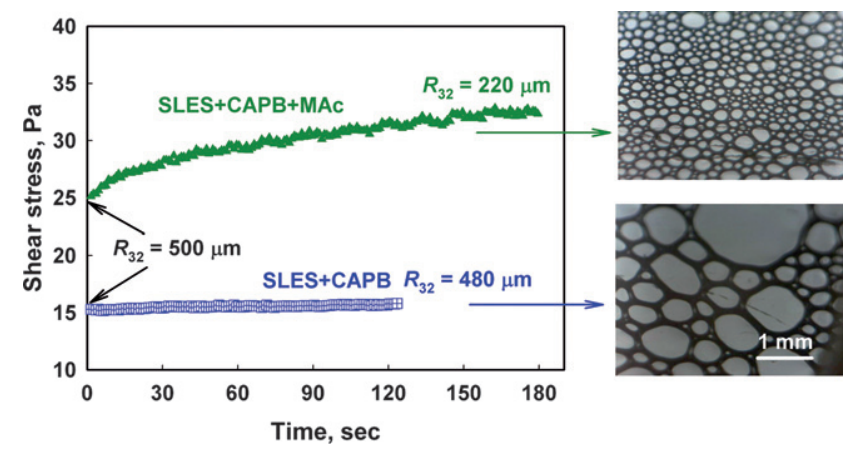

Fig. 12 Measured shear stress of foams, stabilized with different surfactant mixtures as shown in the graph, with initial mean bubble radius, $R_{32} \approx 500 \mu \mathrm{m}$, and maximal bubble radius, $R_{\mathrm{V} 95}=800 \mu \mathrm{m}$. At the end of the shear cycle at $150 \mathrm{~s}^{-1}$ the bubbles in the MAc-containing foam were broken into much smaller bubbles (evidenced also by the observed increase of the shear stress at fixed shear rate), whereas the bubble size distribution in the foam stabilized by SLES + CAPB practically did not change.

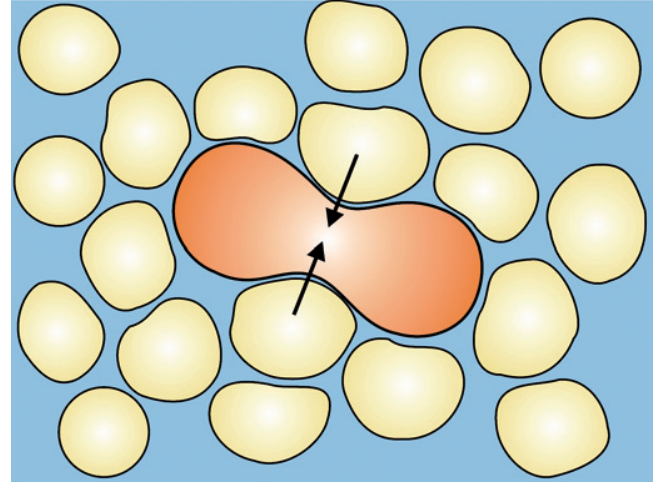

Fig. 13 A schematic presentation of the bubble breakup process in sheared foams. The neighboring bubbles press the thinnest middle part of the elongated "central" bubble, thus creating capillary instability and facilitating the breakup process. This "structural" effect is caused by the close confinement of the bubbles in foams and leads to much lower critical capillary number for breakup than that for single bubbles, sheared in Newtonian liquids (hence, this phenomenon is called "structure-induced capillary instability"). ${ }^{21}$

foams, only when the applied average shear stress, $\tilde{\tau} \equiv(\tau R / \sigma)$ (scaled with the capillary pressure of the bubble) becomes higher than a well defined value, $\tilde{\tau}_{C R} \approx 0.40 \pm 0.02$. Remarkably, this critical dimensionless stress for bubble breakup was found experimentally to be independent of the type of surfactant used, solution viscosity, and bubble volume fraction (varied between 92 and $98 \%$ ). In other words, $\tilde{\tau}_{C R}$ appears as a universal dimensionless parameter for bubble breakup in foams stabilized by surfactants. It is worthwhile noting that the experimentally determined value, $\tilde{\tau}_{C R} \approx 0.40$, is about two orders of magnitude lower than the critical stress, $\tilde{\tau}_{C R} \approx 25$, for breakup of single bubbles $^{110,111}$ in a sheared Newtonian liquid (with viscosity equal to the effective foam viscosity at the same shear rate). This low value of the critical stress in foams was explained with the strong interaction between neighbouring bubbles, which facilitates the appearance of capillary instability of the breaking bubble, with subsequent subdivision into smaller bubbles, see Fig. 13 (this phenomenon was termed "structure-induced capillary instability" of breaking bubbles). ${ }^{21}$

Let us explain now in quantitative terms the effect of surfactant type on the size of formed bubbles in these experiments. As described in section 4 , the viscous stress in foams stabilized by LSM surfactants is determined by the friction inside the foam films only, $\tau_{V}=\tau_{V F}$ (see eqn (5)). On the other hand, when HSM surfactants are used, the shear stress includes also the contribution of surface dissipation, $\tau_{V}=\tau_{V F}+\tau_{V S}$ (see eqn (7)). Upon shear, the radius of the largest bubbles which could survive without braking, $R_{\mathrm{MAX}}$, can be found from the critical stress, $\tilde{\tau}_{C R}$, as follows:

$$
R_{M A X} \approx \frac{\tilde{\tau}_{C R} R_{32}}{\tilde{\tau}_{0}+\tilde{\tau}_{V F}+\tilde{\tau}_{V S}}
$$

where $\tilde{\tau}_{0}=\tau_{0} R_{32} / \sigma$ is the dimensionless yield stress. If the initial foams have similar mean bubble size, $R_{32}$, and similar bubble polydispersity, the largest bubbles in the foam stabilized by HSM surfactants could break at a given shear rate due to the significant contribution of $\tilde{\tau}_{V S}$ in the denominator of eqn (15), whereas 
similar in size bubbles could survive without breakup in the LSM systems, for which $\tilde{\tau}_{V S}$ is negligible. Therefore, at given shear rate, $R_{\mathrm{MAX}}$ will be smaller for foams stabilized by HSM surfactants, as compared to foams stabilized by LSM surfactants, just as observed experimentally.

On this basis, one can easily explain the experimental results shown in Fig. 12. The estimate of the maximum bubble radius by eqn (15) predicts $R_{\mathrm{MAX}} \approx 800 \mu \mathrm{m}$ for SLES + CAPB stabilized foam $\left(\tilde{\tau}_{V F}=0.24\right)$ and $R_{\mathrm{MAX}} \approx 460 \mu \mathrm{m}$ for SLES + Betaine + MAc stabilized foam $\left(\tilde{\tau}_{V F}=0.27, \tilde{\tau}_{V S}=0.15\right)$, which explains why no bubble breakup is observed in SLES + CAPB foams, while fast bubble breakup occurs in MAc-containing foam. Note also that the dimensionless stress $\tilde{\tau}_{V F}$ decreases with the bubble breakup in the latter system (from 0.27 to 0.185 ). Therefore, the maximal radius of the stable bubbles at the end of the experiment with this foam is estimated from eqn (15) to be $R_{\mathrm{MAX}} \approx 260 \mu \mathrm{m}$, which is in a reasonably good agreement with the experimental result, $R_{\mathrm{V} 95} \approx 300 \mu \mathrm{m}$.

Note that all contributions to the shear stress in eqn (15) (viz. $\tau_{0}, \tilde{\tau}_{V F}$, and $\left.\tilde{\tau}_{V S}\right)$ increase with $\Phi$. As a result, eqn (15) predicts that the ratio $R_{\mathrm{MAX}} / R_{32}$ (i.e. foam polydispersity) should also decrease with the increase of $\Phi-$ a nontrivial prediction which was confirmed experimentally. ${ }^{21}$

In conclusion, the effect of surfactant type on the mean bubble size is explained through the effect of surfactants on the shear stress in flowing foams. The experimental data and their analysis reveal an interesting interplay between the shear stress, mean bubble size, and bubble polydispersity which deserves further detailed studies.

\section{Conclusions}

The major aim of this review is to summarize the accumulated knowledge of the effect of surfactants on viscous friction in sheared foams. The experimental results demonstrate convincingly that two qualitatively different classes of surfactants could be distinguished (section 2): the first class is represented by typical synthetic surfactants (SDS, SLES, CAPB), which are characterised by low surface dilatational modulus, $E_{\mathrm{D}}<30 \mathrm{mN} / \mathrm{m}$, (LSM surfactants) and fast relaxation of the surface tension after a rapid perturbation of surface area. The second class of surfactants exhibits high surface modulus, $E_{\mathrm{D}}>100 \mathrm{mN} / \mathrm{m}$ (HSM surfactants), and relatively slow relaxation of the surface tension. Typical examples for this class are the sodium and potassium salts of fatty acids (alkylcarboxylic acids), such as lauric and myristic acids.

With respect to foam rheology, the HSM surfactants lead to significantly higher viscous stress and different scaling laws of the shear stress vs. shear rate (under otherwise equivalent conditions), as compared to LSM surfactants.

The reasons for the observed differences between these two classes of surfactants are explained by the developed theoretical models for the viscous dissipation of energy in foams (sections 4 and 5): for the foam-wall friction, the different surface mobility of the bubbles is decisive - the surfaces of bubbles stabilized by LSM surfactants behave as tangentially mobile, whereas the surfaces of bubbles stabilized by HSM surfactants behave as tangentially immobile. The different surface mobility results in different boundary conditions for the liquid flow in the wetting films formed between the boundary bubbles and the confining solid wall, with concomitant difference in the bubble-wall viscous stress. The scaling laws ( $m=2 / 3$ for mobile surfaces, and $m=1 / 2$ for immobile surfaces at sufficiently large films) emerge from the theoretical analysis of these two types of systems.

For the friction inside sheared foam, the main difference comes from an additional contribution into the dissipated energy in the case of HSM surfactants - namely, the energy dissipation inside the adsorption layer of HSM surfactants is significant, due to the very high surface viscosity of the respective adsorption layers. In contrast, this contribution is negligible for the LSM surfactants, as compared to the viscous friction inside the foam films, formed between neighbouring bubbles in flowing foam. The viscous friction in the films scales with the capillary number of power $n=1 / 2$ (shown both experimentally and theoretically), whereas the surface dissipation scales with the shear rate (not with the capillary number) of power $n \approx 0.18 \pm 0.02$, experimental fact, which still lacks a theoretical explanation.

This deeper understanding of the role of surface properties in foam rheology has allowed us to propose a class of surfactant mixtures combining the advantages of both types of surfactants (section 3), as well as to explain the effect of surfactant type on the process of bubble breakup in sheared foams (section 6).

Let us note at the end that the dynamic properties of surfactant adsorption layers could affect virtually all dynamic phenomena in foams, such as oscillatory and unidirectional deformations, Ostwald ripening, film thinning, non-homogeneous flow at low shear rates, etc. It is important to note that a detailed theoretical analysis, verified by experimental results, is always needed to identify the concrete characteristics of the adsorption layers (dilatational or shear elasticity and viscosity, rates of adsorption and desorption, rate of surface tension relaxation, etc.) which are responsible for the observed differences in specific foam property. From this viewpoint, further systematic theoretical and experimental efforts are very much needed to understand the actual physicochemical mechanisms (and the respective governing factors) of all basic dynamic processes in foams.

Another interesting direction for future systematic studies could be to analyze the observed effects of surfactants from the viewpoint of the shape of the surfactant molecules, which have been extensively studied in relation to micelle and liquid-crystal phase formation in solutions. The results from these studies are often analyzed by considering the so-called "packing parameter" of the surfactant molecules. ${ }^{112,113}$ It would be of interest to check systematically whether the values of the packing parameters, explaining the bulk aggregation behaviour of surfactants, could be related to the properties of the surfactant adsorption layers (and to the resulting dynamic properties of foams). To the best of our knowledge, no such attempt has been made so far in literature.

\section{Acknowledgements}

This study was supported by Unilever R\&D, Trumbull, USA, and is closely related to the activity of COST P21 Action "Physics of Droplets" of the EU. It was partially funded by project No. 202 in the "Rila-4" program of the National Science Fund of Bulgaria. The useful discussions with Prof. M. van Hecke and Dr G. Katgert from Leiden University (who provided Dawn sample 
used in the study) and with Dr M. Vethamuthu from Unilever R\&D USA are gratefully acknowledged.

\section{References}

1 H. M. Princen, The structure, mechanics, and rheology of concentrated emulsions and fluid foams, in Encyclopedia of Emulsion Technology, ed. J. Sjöblom, Marcel Dekker, New York, 2001 , ch. 11 , p. 243.

2 D. Weaire, The rheology of foam, Curr. Opin. Colloid Interface Sci., 2008, 13, 171.

3 D. Weaire and W. Drenckhan, Structure and dynamics of confined foams: A review of recent progress, Adv. Colloid Interface Sci., 2008, 137, 20.

4 R. Höhler and S. Cohen-Addad, Rheology of liquid foam, J. Phys: Condens. Matter, 2005, 17, R1041.

5 D. Langevin, Aqueous foams: A field of investigation at the Frontier between chemistry and physics, ChemPhysChem, 2008, 9, 510.

6 A. M. Kraynik, Foam flows, Ann. Rev. Fluid Mech., 1988, 20, 325.

7 H. M. Princen, Rheology of foams and highly concentrated emulsions: I. Elastic properties and yield stress of a cylindrical model system, J. Colloid Interface Sci., 1983, 91, 160.

8 H. M. Princen, Rheology of foams and highly concentrated emulsions. II. Experimental study of the yield stress and wall effects for concentrated oil-in-water emulsions, J. Colloid Interface Sci. $1985, \mathbf{1 0 5}, 150$.

9 H. M. Princen and A. D. Kiss, Rheology of foams and highly concentrated emulsions: IV. An experimental study of the shear viscosity and yield stress of concentrated emulsions, J. Colloid Interface Sci., 1989, 128, 176.

10 A. Scheludko, Thin liquid films, Adv. Colloid Interface Sci., 1967, 1, 391.

11 I. B. Ivanov, Effect of surface mobility on the dynamic behavior of thin liquid films, Pure Applied Chem., 1980, 52, 1241.

12 D. S. Valkovska, K. D. Danov and I. B. Ivanov, Surfactants role on the deformation of colliding small bubbles, Coll. Surf. A, 1999, 156, 547.

13 K. D. Danov, D. S. Valkovska and I. B. Ivanov, Effect of Surfactants on the Film Drainage, J. Colloid Interface Sci., 1999, 211, 291.

14 S. D. Stoyanov and N. D. Denkov, Role of Surface Diffusion for the Drainage and Hydrodynamic Stability of Thin Liquid Films, Langmuir, 2001, 17, 1150.

15 P. A. Kralchevsky, K. D. Danov, N. D. Denkov, in Handbook of surface and colloid chemistry; ed. K. S. Birdi, CRC, Press LLS, Boca Raton, 1997, ch. 11.

16 N. D. Denkov, S. Tcholakova, K. Golemanov, K. P. Ananthapadmanabhan and A. Lips, Viscous friction in foams and concentrated emulsions under steady shear, Phys. Rev. Lett., 2008, 100, 138301.

17 S. Tcholakova, N. D. Denkov, K. Golemanov, K. P. Ananthapadmanabhan and A. Lips, Theoretical model of viscous friction inside steadily sheared foams and concentrated emulsions, Phys. Rev. E, 2008, 78, 011405.

18 N. D. Denkov, S. Tcholakova, K. Golemanov and A. Lips, Jamming in sheared foams and emulsions, explained by critical instability of the films between neighboring bubbles and drops, Phys. Rev. Lett., 2009, submitted.

19 F. Rouyer, S. Cohen-Addad, M. Vignes-Adler and R. Hoehler, Dynamics of yielding observed in a three-dimensional aqueous dry foam, Phys. Rev. E, 2003, 67, 021405.

20 S. Besson, G. Debregeas, S. Cohen-Addad and R. Hohler, Dissipation in a sheared foam: from bubble adhesion to foam rheology, Phys. Rev. Lett, 2008, 101, 214504.

$21 \mathrm{~K}$. Golemanov, S. Tcholakova, N. D. Denkov, K. P. Ananthapadmanabhan and A. Lips, Breakup of bubbles and drops in steadily sheared foams and concentrated emulsions, Phys. Rev. E, 2008, 78, 051405.

22 D. Exerowa, P. M. Kruglyakov, Foams and Foam Films: Theory, Experiment, Application, Elsevier, Amsterdam, 1998.

23 N. D. Denkov, V. Subraminian, D. Gurovich and A. Lips, Wall slip and viscous dissipation in sheared foams: Effect of surface mobility, Colloids Surf. A, 2005, 263, 129.
24 K. Golemanov, N. D. Denkov, S. Tcholakova, M. Vethamuthu and A. Lips, Surfactant mixtures for control of bubble surface mobility in foam studies, Langmuir, 2008, 24, 9956.

25 S. Marze, D. Langevin and A. Saint-Jalmes, Aqueous foam slip and shear regimes determined by rheometry and multiple light scattering, J. Rheol., 2008, 52, 1091.

26 K. J. Mysels, K. Shinoda, S. Frankel, Soap Films, 1959, Pergamon, Elmsford, NY.

27 A. Prins and F. van Voorst Vader, Proc. 6th Int. Congr. Surf. Act. Subst. (Zurich), 1972, 441.

28 J. Lucassen, in Anionic Surfactants: Physical Chemistry of Surfactant Action, ed. E. H. Lucassen-Reynders, Dekker, New York, 1981, pp. 11.

29 J. L. Joye, G. J. Hirasaki and C. A. Miller, Asymmetric Drainage in Foam Films, Langmuir, 1994, 10, 3174.

30 T. T. Traykov, E. D. Manev and I. B. Ivanov, Hydrodynamics of thin liquid films. Experimental investigation of the effect of surfactants on the drainage of emulsion films, Int. J. Multiphase Flow, 1977, 3, 485.

31 N. D. Denkov, P. Cooper and J.-Y. Martin, Mechanisms of Action of Mixed Solid-Liquid Antifoams. 1. Dynamics of Foam Film Rupture, Langmuir, 1999, 15, 8514.

32 D. Langevin, Influence of interfacial rheology on foam and emulsion properties, Adv. Colloid Interface Sci., 2000, 88, 209.

33 D. Weaire, S. Hutzler, G. Verbist and E. Peters, A review of foam drainage, Adv. Chem. Phys., 1997, 102, 315.

34 A. Saint-Jalmes, Physical chemistry in foam drainage and coarsening, Soft Matter, 2005, 2, 836.

35 H. A. Stone, S. A. Koehler, S. Hilgenfeldt and M. Durand, Perspectives on foam drainage and the influence of interfacial rheology, J. Phys.: Condens. Matter, 2003, 15, S283.

36 G. Verbist, D. Weaire and A. M. Kraynik, The foam drainage equation, J. Phys. Cond. Matter, 1996, 8, 3715.

37 S. Stoyanov, C. Dushkin, D. Langevin, D. Weaire and G. Verbist, Effect of the rheology on foam drainage, Langmuir, 1998, 14, 4663.

38 S. A. Koehler, S. Hilgenfeldt and H. A. Stone, Liquid Flow through aqueous foams: The node-dominated foam drainage equation, Phys. Rev. Lett., 1999, 82, 4232.

39 S. A. Koehler, S. Hilgenfeldt and H. A. Stone, A Generalized View of Foam Drainage: Experiment and Theory, Langmuir, 2000, 16, 6327.

40 S. A. Koehler, S. Hilgenfeldt, E. R. Weeks and H. A. Stone, Drainage of single Plateau borders: Direct observation of rigid and mobile interfaces, Phys. Rev. E, 2002, 66, 040601.

$41 \mathrm{M}$. Durand and D. Langevin, Physicochemical approach to the theory of foam drainage, Eur. Phys. J. E, 2002, 7, 35.

42 M. Safouane, A. Saint-Jalmes, V. Bergeron and D. Langevin, Viscosity effects in foam drainage: Newtonian and non-newtonian foaming fluids, Eur. Phys. J. E, 2006, 19, 195.

43 A. Saint-Jalmes and D. Langevin, Time evolution of aqueous foams: drainage and coarsening, J. Phys.: Condens. Matter, 2002, 14, 9397.

$44 \mathrm{O}$. Pitois, C. Fritz and M. Vignes-Adler, Liquid drainage through aqueous foam: study of the flow on the bubble scale, J. Colloid Interface Sci., 2005, 282, 458.

45 O. Pitois, C. Fritz and M. Vignes-Adler, Hydrodynamic resistance of a single foam channel, Colloids Surf. A, 2005, 261, 109.

46 N. D. Denkov, S. Tcholakova, K. Golemanov, V. Subramanian and A. Lips, Foam-wall friction: Effect of air volume fraction for tangentially immobile bubble surface, Colloids Surf. A, 2006, 282$283,329$.

47 M. Durand and H. A. Stone, Relaxation time of the topological T1 process in a two-dimensional foam, Phys. Rev. Lett., 2006, 97, 226101.

48 R. Soller and S. A. Koehler, Rheology of Steady-State Draining Foams, Phys. Rev. Lett., 2008, 100, 208301.

49 S. Larmignat, D. Vanderpool, H. K. Lai and L. Pilon, Rheology of colloidal gas aphrons (microfoams), Colloid Surf. A, 2008, 322, 199.

50 J. Zhao, S. Pillai and L. Pilon, Rheology of microfoams made from ionic and non-ionic surfactant solutions, Colloid Surf. A, 2009, in press.

51 A. M. Kraynik and D. A. Reinelt. Microrheology of Random Polydisperse Foam, XIVth International Congress on Rheology, 2004, Seoul, Korea.

52 T. G. Mason, J. Bibette and D. A. Weitz, Yielding and flow of monodisperse emulsions, J. Colloid Interface Sci., 1996, 179, 439. 
53 T. G. Mason, J. Bibette and D. A. Weitz, Elasticity of compressed emulsions, Phys. Rev. Lett., 1999, 75, 2051.

$54 \mathrm{~L}$. Becu, S. Manneville and A. Colin, Yielding and flow in adhesive and nonadhesive concentrated emulsions, Rhys. Rev. Lett., 2006, 96, 138302.

55 G. Ovarlez, S. Rodts, A. Ragouilliaux, P. Coussot, J. Goyon and A. Colin, Wide-gap Couette flows of dense emulsions: Local concentration measurements, and comparison between macroscopic and local constitutive law measurements through magnetic resonance imaging, Rhys. Rev. E, 2008, 78, 036307.

56 S. Rodts, J. C. Baudez and P. Coussot, From "discrete" to "continuum" flow in foams, Europhys. Lett., 2005, 69, 636.

57 Y. Otsubo and R. K. Prud'homme, Rheology of oil-in-water emulsions, Rheol Acta, 1994, 33, 29.

58 Y. Otsubo and R. K. Prud'homme, Effect of drop size distribution on the flow behavior of oil-in-water emulsions, Rheol. Acta, 1994, 33, 303.

59 Y. Shen, M. L. Longo and R. L. Powell, Stability and rheological behavior of concentrated monodisperse food emulsifier coated microbubble suspensions, J. Colloid Interface Sci., 2008, 327, 204.

60 F. P. Bretherton, J. Fluid Mech., 1961, 10, 166.

61 L. W. Schwartz, H. M. Princen and A. D. Kiss, J. Fluid Mech., 1986, 172, 259.

62 G. J. Hirasaki and J. B. Lawson, Mechanisms of Foam Flow in Porous Media: Apparent Viscosity in Smooth Capillaries, Soc. Petroleum Eng. J., 1985, 25, 176.

63 J. Ratulowski and H. C. Chang, J. Fluid Mech., 1990, 210, 303.

64 H. Wong, C. J. Radke and S. Morris, Motion of long bubbles in polygonal capillaries. Part 1. Thin films, J. Fluid Mech., 1995, 292, 71.

65 H. Wong, C. J. Radke and S. Morris, Motion of long bubbles in polygonal capillaries. Part 2. Drag, fluid pressure and fluid flow, $J$. Fluid Mech., 1995, 292, 95.

66 I. Cantat, N. Kern and R. Delannay, Europhys. Lett., 2004, 65, 726.

67 A. Saugey, W. Drenkhan and D. Weaire, Phys. Fluids, 2006, 18, 053101.

$68 \mathrm{X}$. Wen, J. Lauterbach and E. I. Franses, Surface densities of adsorbed layers of aqueous sodium myristate inferred from surface tension and infrared reflection absorption spectroscopy, Langmuir, 2000, 16, 6987-6994.

69 P. A. Kralchevsky, K. D. Danov, C. I. Pishmanova, S. D. Kralchevska, N. C. Christov, K. P. Ananthapadmanabhan and A. Lips, Effect of the precipitation of neutral-soap, acid-soap and alkanoic-acid crystallites on the bulk $\mathrm{pH}$ and surface tension of soap solutions, Langmuir, 2007, 23, 3538-3553.

70 P. A. Kralchevsky, M. P. Boneva, K. D. Danov, K. P. Ananthapadmanabhan and A. Lips, Method for analysis of the composition of acid soaps by electrolytic conductivity measurements, J. Colloid Interface Sci., 2008, 327, 169-179.

71 J. Lucassen and M. van den Tempel, Chem. Eng. Sci., 1972, 27, 1283.

72 D. A. Edwards, D. T. Wasan, H. Brenner, Interfacial Transport Processes and Rheology, Butterworth-Heinemann, Boston, 1991.

73 E. M. Freer, K. Sub Yim, G. G. Fuller and C. J. Radke, Shear and dilatational relaxation mechanisms of globular and flexible proteins at the hexadecane/water interface, Langmuir, 2004, 20, 10159-10167.

74 S. C. Russev, N. Alexandrov, K. G. Marinova, K. D. Danov, N. D. Denkov, L. Lyutov, V. Vulchev and C. Bilke-Krause, Review of Scientific Instruments, 2008, 79, 104102.

75 J. G. Petrov, Th. Pfohl and H. Mohwald, Ellipsometric chain length dependence of fatty acid langmuir monolayers. A heads-and-tails model, J. Phys. Chem. B, 1999, 103, 3417-3424.

76 J. Janiaud, D. Weaire and S. Hutzler, Phys. Rev. Lett., 2006, 97, 038302.

77 V. J. Langlois, S. Hutzler and Denis Weaire, Rheological properties of the soft-disk model of two-dimensional foams, Phys. Rev. E, 2008, 78, 021401 .

78 D. Weaire, S. Hutzler, V. J. Langlois and R. J. Clancy, Velocity dependence of shear localisation in a 2D foam, Philos. Mag. Lett., 2008, 88, 387.

79 G. Katgert, M. E. Möbius and M. van Hecke, Phys. Rev. Lett, 2008, 101, 058301.

80 M. E. Mobius, G. Katgert and Martin van Hecke, Relaxation and flow in linearly sheared two-dimensional foams, arXiv:0811.0534v1 [cond-mat.soft] 4 Nov 2008.

81 G. Katgert, PhD Thesis, Leiden University, 2008.
82 J. Ratulowski and H.-C. Chang, Marangoni effects of trace impurities on the motion of long gas bubbles in capillaries, Journal of Fluid Mechanics, 1990, 210, 303-328.

83 R. Palaparthi, D. T. Papageorgiou and C. Maldarelli, Theory and experiments on the stagnant cap regime in the motion of spherical surfactant-laden bubbles, J. Fluid Mech., 2006, 559, $1-44$.

84 K. J. Stebe and C. Maldarelli, Remobilizing surfactant retarded fluid particle interfaces. II. Controlling the surface mobility at interfaces of solutions containing surface active components, J. Colloid Interface Sci., 1994, 163, 177-189.

85 S. Tcholakova, N. Denkov, K. Golemanov, V. Subramanian, A. Lips, Effect of surface mobility on the bubble-wall friction, 6th European Conference of Foams, Emulsions and Applications, 2-6 July, 2006, Potsdam, Germany, poster.

86 V. Carrier, S. Destouesse and A. Colin, Foam drainage: A film contribution?, Rhys Rev E, 2002, 65, 061404.

87 Y. Saiki, C. A. Prestidge and R. G. Horn, Effects of droplet deformability on emulsion rheology, Colloids Surf. A, 2007, 299, $65-72$.

88 Y. Saiki, R. G. Horn and C. A. Prestidge, Droplet structure instability in concentrated emulsions, J. Colloid Interface Sci., 2008, 320, 569-574.

89 Y. Saiki, R. G. Horn and C. A. Prestidge, Rheological transition of concentrated emulsions during successive shearing cycles, J. Colloid Interface Sci., 2008, 327, 440.

90 S. P. Meeker, R. T. Bonnecaze and M. Cloitre, Slip and flow in soft particle pastes, Phys. Rev. Lett., 2004, 92, 198302.

91 S. Meeker, R. T. Bonnecaze and M. Cloitre, Slip and flow in pastes of soft particles: direct observations and rheology, J. Rheol., 2004, 48, 1295.

92 J. R. Seth, M. Cloitre and R. T. Bonnecaze, Influence of short-range forces on wall-slip in microgelpastes, J. Rheol., 2008, 52, 1241.

93 C. Raufaste, B. Dollet, S. Cox, Y. Jiang and F. Graner, Yield drag in a two-dimensional flow of foam around a circular obstacle: Effect of fluid fraction, Euro. Phys. J. E, 2007, 23, 217.

94 B. Dollet, M. Durth and F. Graner, Flow of foam past an elliptical obstacle, Phys. Rev. E, 2006, 73, 061404.

95 J. Lauridsen, G. Chanan and M. Dennin, Velocity profiles in slowly sheared bubble rafts, Phys. Rev. Lett., 2004, 93, 018303.

96 C. Gilbreth, S. Sullivan and M. Dennin, Flow transitions in twodimensional foams, Phys. Rev. E, 2006, 74, 051406.

97 G. Debregeas, H. Tabuteau and J.-M. di Meglio, Deformation and flow of a two-dimensional foam under continuous shear, Phys. Rev. Lett., 2001, 87, 178305.

98 E. Janiaud, D. Weaire and S. Hutzler, Two-dimensional foam rheology with viscous drag, Phys. Rev. Lett., 2006, 97, 038302.

99 S. J. Cox and E. L. Whittick, Shear modulus of two-dimensional foams: The effect of area dispersity and disorder, Euro. Phys. J. E, 2006, 21, 49 .

100 B. Dollet and F. Graner, Two-dimensional flow of foam around a circular obstacle: local measurements of elasticity, plasticity and flow, J. Fluid Mech., 2007, 585, 181.

101 D. J. Durian, Phys. Rev. Lett., 1995, 75, 4780.

102 D. J. Durian, Phys. Rev. E, 1997, 55, 1739.

103 S. I. Karakashev, A. V. Nguyen, E. Manev and C. M. Phan, Surface foam film waves studied with high-speed linescan camera, Colloids Surf. A, 2005, 262, 23-32.

104 E. Manev, Effect of thickness non-homogeneity on the kinetic behaviour of microscopic foam films, J. Dispersion Sci. Tech., 1997, 18, 769-788.

105 F. Rouyer, S. Cohen-Addad, R. Heohler, P. Sollich and S. M. Fielding, The large amplitude oscillatory strain response of aqueous foam: Strain localization and full stress Fourier spectrum, Eur. Phys. J. E, 2008, 27, 309-321.

106 A. D. Gopal and D. J. Durian, Shear-induced "melting" of an aqueous foam, J. Colloid Interface Sci., 1999, 213, 169.

107 H. T. Chen and S. Middleman, Drop size distribution in agitated liquid-liquid systems, AIChE Journal, 1967, 13, 989.

108 J. T. Davies, Drop sizes of emulsions related to turbulent energy dissipation rates, Chem. Eng. Sci., 1985, 40, 839.

109 N. Vankova, S. Tcholakova, N. D. Denkov, I. B. Ivanov, V. Vulchev and T. Danner, Emulsification in turbulent flow 1. Mean and maximum drop diameters in inertial and viscous regimes, $J$. Colloid Interface Sci., 2007, 312, 363-380. 
$110 \mathrm{~J}$. M. Rallison, The deformation of small viscous drops and bubbles in shear flows, Annu. Rev. Fluid Mech., 1984, 16, 45.

111 E. J. Hinch and A. Acrivos, Long slender drops in a simple shear flow, J. Fluid Mech., 1980, 98, 305.
112 J. N. Israelachvili, Intermolecular and Surface Forces, Academic Press, New York, 2nd edn, 1992.

113 D. F. Evans, H. Wennerstoem, The Colloidal Domain, Wiley-VCH, New York, 2nd edn, 1999. 